\title{
Generalized FEM for Homogenization Problems
}

\section{Report}

Author(s):

Matache, Ana-Maria; Schwab, Christoph

Publication date:

2001-04

Permanent link:

https://doi.org/10.3929/ethz-a-004289348

Rights / license:

In Copyright - Non-Commercial Use Permitted

Originally published in:

SAM Research Report 2001-03 


\title{
Generalized FEM for Homogenization Problems ${ }^{\star}$
}

\author{
A.-M. Matache ${ }^{\star}$ and C. Schwab
}

Research Report No. 2001-03

April 2001

Seminar für Angewandte Mathematik Eidgenössische Technische Hochschule

CH-8092 Zürich

Switzerland

\footnotetext{
* Research supported by the Swiss National Science Foundation under Project Number BBW 21-58754.99 and partially supported by the DFG Schwerpunktprogramm 'Multiple Scale Problems'

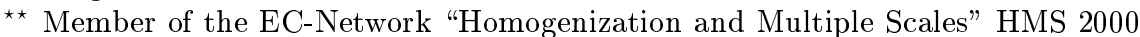




\title{
Generalized FEM for Homogenization Problems ${ }^{\star}$
}

\author{
A.-M. Matache ${ }^{\star \star}$ and C. Schwab
}

Seminar für Angewandte Mathematik

Eidgenössische Technische Hochschule

CH-8092 Zürich

Switzerland

Research Report No. 2001-03

April 2001

\begin{abstract}
We introduce the concept of generalized Finite Element Method (gFEM) for the numerical treatment of homogenization problems. These problems are characterized by highly oscillatory periodic (or patchwise periodic) pattern in the coefficients of the differential equation and their solutions exhibit a multiple scale behavior: a macroscopic behavior superposed with local characteristics at micro length scales. The gFEM is based on two-scale FE spaces that are obtained by augmenting the standard polynomial FE spaces with problem dependent, non-polynomial micro shape functions that reflect the oscillatory behavior of the solution. Our choice of micro-scale shape functions is motivated by a new class of representations formulas for the solutions on unbounded domains, which are based on an assumption of scale separation and generalized Fourier inversion integrals. Under analiticity assumptions on the input data, gFEM converges robustly and exponentially. The micro-shape functions obtained from the representation formula are solutions to suitable unit-cell problems. In the gFEM, they are obtained in the start-up phase of the calculations by solving numerically these unit-cell problems.

Different choices of the micro shape functions are possible within the framework of the gFEM and we also analyze gFEM based on micro shape functions obtained from the theory of Bloch waves. Numerical results for one-dimensional and twodimensional problems corroborate our theoretical results.
\end{abstract}

\footnotetext{
* Research supported by the Swiss National Science Foundation under Project Number BBW 21-58754.99 and partially supported by the DFG Schwerpunktprogramm 'Multiple Scale Problems'

** Member of the EC-Network "Homogenization and Multiple Scales" HMS 2000
} 


\section{Introduction}

We consider the Finite Element approximation of the classical elliptic homogenization problem:

$$
\begin{gathered}
L^{\varepsilon}\left(x, \partial_{x}\right) u^{\varepsilon}:=-\nabla \cdot\left(a^{\varepsilon}(x) \nabla u^{\varepsilon}\right)+a_{0}^{\varepsilon}(x) u^{\varepsilon}=f \quad \text { in } \Omega^{\varepsilon}, \\
B u^{\varepsilon}=0 \quad \text { on } \partial \Omega^{\varepsilon} .
\end{gathered}
$$

Here, the set $\Omega^{\varepsilon}=\Omega_{\infty}^{\varepsilon} \cap \Omega$ is defined as the intersection of a bounded Lipschitz domain $\Omega$ and the infinite periodic lattice of width $\varepsilon$ given by

$$
\Omega_{\infty}^{\varepsilon}:=\varepsilon \Omega_{\infty}, \quad \Omega_{\infty}:=\bigcup_{k \in \mathbb{Z}^{n}}(2 \pi k+\widehat{Q}),
$$

and $\widehat{Q} \subset \widehat{Y}:=[-\pi, \pi]^{n}, \partial \widehat{Q}$ Lipschitz, is the unit-cell. We assume that $\Omega_{\infty}^{\varepsilon}$ is connected. The case when $\widehat{Q} \neq[-\pi, \pi]^{n}$ and the interior boundary of $\widehat{Q}$ is merely piecewise smooth models problems where the material has holes or rigid inclusions. If $\widehat{Q}=[-\pi, \pi]^{n}$, then $\Omega^{\varepsilon}=\Omega$. Under these assumptions, the domain $\Omega^{\varepsilon}$ is a bounded, connected subset of $\mathbb{R}^{n}$ with boundary $\partial \Omega^{\varepsilon}$ and associated boundary operator $B$ which may be either the trace operator or the conormal derivative operator. In electrostatics, for example, $u^{\varepsilon}$ is the electrostatic potential, $a^{\varepsilon}$ represents the dielectric permittivity matrix and the parameter $\varepsilon$ describes the size of the material heterogeneities. Since $\varepsilon$ is small compared to the size of $\Omega$, we have a problem with multiple scales: a macroscopic one (the size of the sample $\Omega$ ) and a microscopic one (the size of the heterogeneities, given by $\varepsilon$ ). The heterogeneities are assumed to be periodically distributed, i.e., $a^{\varepsilon}(x)=a(x / \varepsilon), a_{0}^{\varepsilon}(x)=a_{0}(x / \varepsilon)$ and the coefficients $a(\cdot):=\left(a_{p q}(\cdot)\right)_{p q} \in L^{\infty}\left(\mathbb{R}^{n}\right)_{\mathrm{sym}}^{n \times n}, a_{0}(\cdot) \in L^{\infty}\left(\mathbb{R}^{n}\right)$ are $2 \pi$-periodic functions in each variable. It is also assumed that there exist positive constants $\gamma_{0}, \gamma_{1}>0$ such that

$$
\begin{aligned}
& \gamma_{0} \eta^{\top} \eta \leq \sum_{p, q=1}^{n} a_{p q}(\xi) \eta_{p} \eta_{q} \leq \gamma_{1} \eta^{\top} \eta, \forall \eta \in \mathbb{R}^{n}, \text { for a.e. } \xi \in \mathbb{R}^{n}, \\
& \gamma_{0} \leq a_{0}(\xi) \leq \gamma_{1} \text {, for a.e. } \xi \in \mathbb{R}^{n} .
\end{aligned}
$$

Under these assumptions, problem (1), (2) admits a unique solution.

In this work, we consider numerical solution of (1), (2) by the Finite Element Method (FEM). After discussing the scale-resolution requirement for standard FEM, we design in Section 2 generalized FEM which allow to resolve the $\varepsilon$ scale of the solution without mesh refinement down to scale $\varepsilon$. These generalized FEM are built from a macroscopic mesh together with a 'microscopic' FE-space $\mathcal{M}_{\varepsilon}^{\mu}$ of $\varepsilon$-periodic functions attached to each macroscopic degree of freedom. Several possible choices of $\mathcal{M}_{\varepsilon}^{\mu}$ are conceivable and the performance of the generalized FEM strongly depends on the choice of the micro-space. The specific choice of $\mathcal{M}_{\varepsilon}^{\mu}$ will be determined by the regularity of the exact solution $u^{\varepsilon}$. From classical homogenization theory, rather complete asymptotic expansions as $\varepsilon \rightarrow 0$ of the exact solution $u^{\varepsilon}$ are available. The profiles in these expansions could, in principle, be used in the construction of $\mathcal{M}_{\varepsilon}^{\mu}$. Here, we shall use a representation of the solution in terms of a (generalized) Fourier integral. This is presented in Section 3 together with a methodology for deriving $\varepsilon$-dependent FE-spaces which allow robust (i.e. uniform in the scale $\varepsilon$ ) exponential convergence to the exact solution. The Fourier representation allows for new two-scale regularity estimates for the solution $u^{\varepsilon}$ which are robust and explicit in $\varepsilon$ (see [28]) and which, in turn, 
lead to the design of generalized FEM which allow for robust and optimal (in terms of the regularity of the data) convergence rates. We present here theoretical results only in an expository fashion, and refer to $[31,32,26,28,29]$ for a detailed analysis.

\section{Generalized FEM}

In this section we present the abstract formulation of the two-scale FEM with generic micro space $\mathcal{M}_{\varepsilon}^{\mu} \subset H_{\text {per }}^{1}(\varepsilon \widehat{Q})$ and discuss implementational aspects of the two-scale FEM. In particular, for macroscopic FE triangulations that are aligned with the periodic structure for which the computation of the element stiffnes matrices can be done with work independent of $\varepsilon$. Specific microspaces $\mathcal{M}_{\varepsilon}^{\mu}$ will be discussed below.

\subsection{The homogenization problem on bounded domains}

We present the ideas for a Dirichlet problem, but emphasize that other boundary conditions may be considered as well. As before, $\Omega$ is a bounded, Lipschitz subdomain of $\mathbb{R}^{n}$. Let $f(x) \in L^{2}(\Omega)$ and let $\varepsilon>0$ fixed. Denote by $u^{\varepsilon}(x) \in H_{0}^{1}(\Omega)$ the solution of the following boundary value problem

$$
\begin{aligned}
L^{\varepsilon}\left(x, \partial / \partial_{x}\right) u^{\varepsilon}(x):=-\nabla \cdot\left(a\left(\frac{x}{\varepsilon}\right) \nabla u^{\varepsilon}(x)\right)+a_{0}\left(\frac{x}{\varepsilon}\right) u^{\varepsilon}(x) & =f(x) \text { in } \Omega \\
\left.u^{\varepsilon}\right|_{\partial \Omega} & =0,
\end{aligned}
$$

with coefficients $a(\cdot) \in L^{\infty}\left(\mathbb{R}^{n}\right)_{\mathrm{sym}}^{n \times n}, a_{0}(\cdot) \in L^{\infty}\left(\mathbb{R}^{n}\right)$ being $2 \pi$-periodic functions and satisfying the strong ellipticity conditions (3)-(4). The finite element method is based on the usual variational formulation of (5):

Find $u^{\varepsilon}(x) \in H_{0}^{1}(\Omega)$ such that $B^{\varepsilon}\left(u^{\varepsilon}, v\right)=(f, v)_{L^{2}(\Omega)} \quad \forall v \in H_{0}^{1}(\Omega)$,

where the bilinear form $B^{\varepsilon}(\cdot, \cdot): H_{0}^{1}(\Omega) \times H_{0}^{1}(\Omega) \rightarrow \mathbb{R}$ is given by

$$
B^{\varepsilon}(u, v):=\int_{\Omega} a\left(\frac{x}{\varepsilon}\right) \nabla u(x) \cdot \nabla v(x)+a_{0}\left(\frac{x}{\varepsilon}\right) u(x) v(x) d x \quad \forall u, v \in H_{0}^{1}(\Omega) .
$$

The finite element method $[12,10]$ is then defined as follows: for a subspace $V_{N} \subset H_{0}^{1}(\Omega)$ of finite dimension $N=\operatorname{dim}\left(V_{N}\right)$, find $u_{N}^{\varepsilon} \in V_{N}$ such that

$$
B^{\varepsilon}\left(u_{N}^{\varepsilon}, v\right)=(f, v)_{L^{2}(\Omega)} \quad \forall v \in V_{N} .
$$

(7) is a linear system of $N$ equations. Selecting a basis $\left\{\varphi_{j}^{N}: j=1, \ldots, N\right\}$ for $V_{N}$, we can expand $u_{N}^{\varepsilon}$ and $v \in V_{N}$ in terms of the shape functions $\left\{\varphi_{j}^{N}\right\}$ as

$$
u_{N}^{\varepsilon}=\sum_{i=1}^{N} u_{i, N}^{\varepsilon} \varphi_{i}^{N}, \quad v=\sum_{j=1}^{N} v_{j, N} \varphi_{j}^{N}
$$

with coefficients $u_{i, N}^{\varepsilon}, v_{j, N} \in \mathbb{R}$. Inserting this into (7) we obtain the linear system for the coefficient vector $\underline{u_{N}^{\varepsilon}}=\left\{u_{i, N}^{\varepsilon}\right\}_{i=1}^{N}$

$$
\underline{A}^{\varepsilon} \underline{u}_{N}^{\varepsilon}=\underline{f}
$$

in which the stiffness matrix $\underline{\underline{A}}^{\varepsilon}$ and the load vector $\underline{f}$ are given by

$$
\underline{\underline{A}}_{i j}^{\varepsilon}=B^{\varepsilon}\left(\varphi_{j}^{N}, \varphi_{i}^{N}\right), \quad \underline{f}_{i}=\left(f, \varphi_{i}^{N}\right)_{L^{2}(\Omega)}, \quad \forall i, j=1, \ldots, N .
$$


By the Lax-Milgram Theorem, there exists a unique weak solution of (7) and the error $e_{N}^{\varepsilon}:=u^{\varepsilon}-u_{N}^{\varepsilon}$ is quasioptimal in the sense that for some $C>0$ independent of $\varepsilon$ it holds [11]

$$
\left\|e_{N}^{\varepsilon}\right\|_{H^{1}(\Omega)} \leq C \inf _{v \in V_{N}}\left\|u^{\varepsilon}-v\right\|_{H^{1}(\Omega)} .
$$

Thus, the error is completely determined by the approximation properties of the space $V_{N}$.

In view of (10) we design the generalized FEM to satisfy the following two criteria: a) the spaces $V_{N}$ should have robust (i.e. independent of $\varepsilon$ ) approximation properties, and b) the linear system (8) can be set up efficiently.

For standard FE spaces, scale resolution, i.e., the discretization of the smallscale problem features with finite elements, is not feasible, even with advanced computing hardware. Even if the right-hand side $f$, the unit-cell $\widehat{Q}$, the domain $\Omega$ and the coefficients $a$ and $a_{0}$ are smooth (i.e., $C^{\infty}$ ), for $\varepsilon / \operatorname{diam}(\Omega) \ll 1$ the solution $u^{\varepsilon}$ exhibits oscillations on the $\varepsilon$-scale which spoil the FE-convergence. To see this assume that $\widehat{Q}=[-\pi, \pi]^{n}$. Then, $\Omega^{\varepsilon}=\Omega$ and there exist positive constants $C=C(\Omega)$ and $C(\alpha)=C(\alpha, \Omega), \alpha \in \mathbb{N}^{n}$, such that

$$
\left\|u^{\varepsilon}\right\|_{L^{2}(\Omega)} \leq C, \quad\left\|D^{\alpha} u^{\varepsilon}\right\|_{L^{2}(\Omega)} \leq C(\alpha) \varepsilon^{1-|\alpha|}, \quad \forall \alpha \in \mathbb{N}^{n},|\alpha|>0 .
$$

Then, denoting by $\mathcal{V}_{\varepsilon}^{N}=\mathcal{V}_{N}=S^{p, 1}\left(\Omega, \mathcal{T}_{H}\right) \subset H^{1}(\Omega)$ the FE space of piecewise polynomials of degree $p \geq 1$ on a quasiuniform mesh $\mathcal{T}_{H}$ of meshwidth $H$, it holds that

$$
\min _{v \in S^{p, 1}\left(\Omega, \mathcal{T}_{H}\right)}\left\|u^{\varepsilon}-v\right\|_{E} \leq C H^{p}\left\|D^{p+1} u\right\|_{L^{2}(\Omega)} \leq C(H / \varepsilon)^{p} .
$$

Since the energy projection is bounded, we also have

$$
\min _{v \in S^{p, 1}\left(\Omega, \mathcal{T}_{H}\right)}\left\|u^{\varepsilon}-v\right\|_{E} \leq\left\|u^{\varepsilon}\right\|_{E} \leq C\|f\|_{L^{2}(\Omega)} .
$$

It follows therefore that the FE error with respect to the FE space $\mathcal{V}_{N}=$ $S^{p, 1}\left(\Omega, \mathcal{T}_{H}\right)$ satisfies the following a-priori bounds

$$
\frac{\left\|u^{\varepsilon}-u_{N}^{\varepsilon}\right\|_{E}}{\left\|u^{\varepsilon}\right\|_{E}} \leq \min \left(1, C(H / \varepsilon)^{p}\right),
$$

with $C=C\left(p, \Omega, f, a, a_{0}\right)>0$ being a constant independent of $\varepsilon$ and $H$. Standard FEM such as piecewise linears on a quasiuniform mesh $\mathcal{T}_{H}$ of size $H$ converge only if $H<\varepsilon$, i.e., if $N=\operatorname{dim} \mathcal{V}_{\varepsilon}^{N}=O\left(\varepsilon^{-n}\right)$ so that the $\varepsilon$-scale is resolved. Moreover, high order FEM $(p>1)$ will essentially not improve the scale resolution.

To overcome this nonrobustness of the standard FEM, we have developed generalized FEM where the size $H$ of the element is larger than the scale $\varepsilon$ of the oscillatory coefficient. The main idea is to replace the polynomials by function spaces that are, in a sense, adapted to the coefficients of the elliptic operator, i.e., information at scale $\varepsilon$ is built into the shape functions.

\subsection{Generalized two-scale FEM}

We consider (1) with homogeneous Dirichlet boundary conditions on $\Gamma_{D}^{\varepsilon}:=$ $\partial \Omega^{\varepsilon} \cap \partial \Omega$ and homogeneous Neumann boundary conditions on the (if nonempty) interior cavity' boundaries $\Gamma_{N}^{\varepsilon}:=\partial \Omega^{\varepsilon} \backslash \Gamma_{D}^{\varepsilon}$

$$
\begin{aligned}
& \left.u^{\varepsilon}\right|_{\Gamma_{D}^{\varepsilon}}=0 \\
& \left.n \cdot\left(a\left(\frac{x}{\varepsilon}\right) \nabla u^{\varepsilon}\right)\right|_{\Gamma_{N}^{\varepsilon}}=0 .
\end{aligned}
$$


The solution $u^{\varepsilon}$ of (1), (11) is an element of the space $H_{\Gamma_{D}^{\varepsilon}}^{1}\left(\Omega^{\varepsilon}\right)$ defined as

$$
H_{\Gamma_{D}^{\varepsilon}}^{1}\left(\Omega^{\varepsilon}\right):=\left\{u \in H^{1}\left(\Omega^{\varepsilon}\right)\left|\gamma_{0} u\right|_{\Gamma_{D}^{\varepsilon}}=0\right\}
$$

where $\gamma_{0}$ here denotes the Dirichlet trace operator. Two-scale FEMs are built from a macro and a micro FE-space. Let $\mathcal{T}$ be a regular mesh in $\Omega$. The macro FE-space $S_{0}^{p}, 1(\Omega, \mathcal{T}) \subset H_{0}^{1}(\Omega)$ is the usual FE space given by

$$
S_{0}^{\underline{p}, 1}(\Omega, \mathcal{T}):=\left\{u \in H_{0}^{1}(\Omega):\left.u\right|_{K} \in S^{p_{K}}(K), \forall K \in \mathcal{T}\right\} .
$$

Here $\underline{p}=\left\{p_{K}\right\}_{K \in \mathcal{T}}$ is a degree vector and $S^{p_{K}}(K)$ denotes the elemental polynomial space in each element. We will refer to $\underline{p}$ as the macro degree and to $\mathcal{T}$ as the macro mesh of the two-scale FEM.

The micro FE-space $\mathcal{M}_{\varepsilon}^{\mu}, \mu \geq 0$, is a generic finite dimensional subspace of $H_{\mathrm{per}}^{1}(\varepsilon \widehat{Q})$ of $\varepsilon$-periodic, oscillatory shape functions

$$
H_{\mathrm{per}}^{1}(\varepsilon \widehat{Q}) \supset \mathcal{M}_{\varepsilon}^{\mu}:=\operatorname{Span}\left\{\phi_{j}\left(\frac{x}{\varepsilon}\right): j=1, \ldots, \mu+1\right\}, \quad \mathcal{M}^{\mu}:=\mathcal{M}_{1}^{\mu},
$$

with $\phi_{j}(y) \in H_{\mathrm{per}}^{1}(\widehat{Q}), \forall j$. A basic assumption on the space $\mathcal{M}_{\varepsilon}^{\mu}$ is that $\mathcal{M}_{\varepsilon}^{0}=\mathcal{M}^{0}=\operatorname{Span}\{1\}$. As in the degree vector $\underline{p}$, we associate with each macroelement $K \in \mathcal{T}$ a micro degree $\mu_{K}$ and we denote by $\underline{\mu}$ the micro degree vector $\underline{\mu}:=\left\{\mu_{K}\right\}_{K \in \mathcal{T}}$. We define the generalized two-scale FE space $S_{0}^{\underline{p}}\left(\Omega, \mathcal{T} ; \mathcal{M} \frac{\mu}{\varepsilon}\right) \subset H_{\Gamma_{D}^{\varepsilon}}^{1}\left(\Omega^{\varepsilon}\right)$ as

$$
\begin{aligned}
S_{0}^{\frac{p}{(}\left(\Omega, \mathcal{T} ; \mathcal{M} \frac{\mu}{\varepsilon}\right)} & =S_{0}^{p, 1}(\Omega, \mathcal{T}) \otimes_{\mathcal{T}} \mathcal{M} \frac{\mu}{\varepsilon} \\
& :=\left\{u \in H_{\Gamma_{D}^{\varepsilon}}^{1}\left(\Omega^{\varepsilon}\right)|u|_{K} \in S^{p_{K}}(K) \otimes \mathcal{M}_{\varepsilon}^{\mu_{K}} \quad \forall K \in \mathcal{T}\right\}
\end{aligned}
$$

If $\mathcal{M}_{\varepsilon}^{\mu}$ itself is a standard FE-space, we will use the notation $S \underline{p}, \underline{\mu}(\Omega, \mathcal{T} ; \varepsilon \widehat{Q}, \varepsilon \widehat{\mathcal{T}})$ for the two grid FE space corresponding to

$$
\mathcal{M}^{\underline{\mu}}=S \frac{\mu}{\operatorname{per}}(\widehat{Q}, \widehat{\mathcal{T}})
$$

the polynomial FE space on the unit-cell of degree $\mu$ on the unit-cell mesh $\widehat{\mathcal{T}}$.

Due to the assumption that $\operatorname{Span}\{1\} \subset \mathcal{M}_{\varepsilon}^{\mu}$ for all $\mu \geq 0$, the macroscopic polynomial space is always a subspace of $S_{0}^{\frac{p}{0}}\left(\Omega, \mathcal{T} ; \mathcal{M} \frac{\mu}{\varepsilon}\right)$. We can refer to $S \frac{p}{0}\left(\Omega, \mathcal{T} ; \mathcal{M} \frac{\mu}{\varepsilon}\right)$ as the generalized FE space and to the FEM based on $S_{0}^{p}\left(\Omega, \mathcal{T} ; \mathcal{M} \frac{\mu}{\varepsilon}\right)$ as generalized two-scale FEM.

We emphasize the flexibility provided by the tensor product construction of (14). Using a macro-mesh $\mathcal{T}$ allows us to represent the geometry as is customary in standard FEM. If we use elements $K \in \mathcal{T}$ of size $O(\varepsilon)$ in a small neighborhood of $\partial \Omega$ and choose there the micro degree $\mu=0$, then the gFEM space reduces to a standard FEM with scale resolution at the boundary. This allows us to enforce boundary conditions directly, i.e. without recourse to the homogenized equation. In the interior of the domain, we choose large elements $K$ and choose there $\mu>0$, i.e. we couple in effect the generalized FEM in the interior to a standard FEM near the boundary.

Remark 1. For domains $\Omega$ with corners, we could also refine the macro mesh $\mathcal{T}$ geometrically toward the corners as is done in the $h p$-FEM for problems with piecewise analytic input data, [37,23]. However, for macro meshes $\mathcal{T}$ 
with strong local refinements, care has to be taken in the selection of $\mu$ : once for $K \in \mathcal{T} \operatorname{diam}(K)=O(\varepsilon)$, the macro mesh locally resolves the fine scale of $u^{\varepsilon}$ and in such elements the micro degree $\mu$ may be reduced (even to $\mu=0$ ) in order to avoid linear dependence.

It might be of interest to use a macro mesh $\mathcal{T}$ with anisotropic elements at the boundary: boundary macro elements abutting at a corner or singularity with size $<\varepsilon$ where the micro shape functions are omitted $\left(\mu_{K}=0\right)$ or elongated elements abutting at the boundary where $\mu_{K}>0$ are necessary to resolve the tangential fine structure of the solution. In that case, the micro space $\mathcal{M}_{\varepsilon}^{\mu}$ should be chosen so as to reflect highly oscillatory character of the solution in the tangential direction. These micro shape functions would have to be derived from appropriate problems on the unit-cell. The interior elements are of size $\gg \varepsilon$ and $\mu_{K}>0$ there, i.e., the micro scales are accounted by the oscillatory shape functions $\phi_{j}(x / \varepsilon), 0<j \leq \mu_{k}$.

\subsection{Implementation of two-scale FEM}

In order to obtain an efficient algorithm it is essential that the element stiffness and mass matrices can be computed in a complexity independent of $\varepsilon$ and to an accuracy which will not compromise the asymptotic convergence rates. Due to the rapid oscillations of the coefficients and of the microshapefunctions, the elemental stiffness matrices on the macro mesh can not be evaluated robustly, i.e., with work independent of $\varepsilon$, by standard quadratures. A remedy is either to resort to massively parallel computation as in $[20,21]$, or to exploit periodicity. It turns out that the macro stiffness and mass matrices can be developed from moments, i.e., integrals with respect to monomial weighted coefficients in the fast variable of the micro scale shape functions on the unit-cell combined with certain lattice summation formulas.

Proposition 2. Let $\varepsilon>0$ and let $\mathcal{M}^{\mu}(\widehat{Q})=\operatorname{Span}\left\{\phi_{i}\right\}_{i=0}^{\mu}$ be any conforming $F E$ discretization of $H_{\mathrm{per}}^{1}(\widehat{Q})$ of dimension $\mu+1$ independent of $\varepsilon$, and assume that the macro mesh $\mathcal{T}_{H}$ is aligned with the periodic microstructure. Then the FEM with respect to the two-scale discretization is

$$
S^{p}\left(\Omega, \mathcal{T}_{H} ; \mathcal{M}_{\varepsilon}^{\mu}(\varepsilon \widehat{Q})\right)
$$

where $\mathcal{M}_{\varepsilon}^{\mu}(\varepsilon \widehat{Q})=\operatorname{Span}\left\{\phi_{i}(x / \varepsilon)\right\}_{i=0}^{\mu}$, can be implemented with computational work independent of $\varepsilon$.

The $\varepsilon$-independence is achieved by judiciously exploiting the periodicity in the fast variable. The stiffness matrix of the generalized FEM can be computed with a fixed number of operations (independent of $\varepsilon$ ) by exploiting the periodicity of the coefficients $a(\cdot), a_{0}(\cdot)$ and that of the special shape functions $\phi_{j}(y)$. We must compute only once integrals of $\phi_{j}(y)$ and their derivatives multiplied by monomials on the unit cell. In order to see this we state the following lemma:

Lemma 3. Assume that $j \in \mathbb{N}, F(\cdot)$ is 1 -periodic and $\varepsilon=1 / M$ with $M \in \mathbb{N}$. Then

$$
\int_{0}^{1} x^{j} F\left(\frac{x}{\varepsilon}\right) d x=\sum_{k=0}^{j} \varepsilon^{j-k}\left(\begin{array}{l}
j \\
k
\end{array}\right) S(M, k) \int_{0}^{1} y^{j-k} F(y) d y
$$

where

$$
S(M, k)=\frac{1}{M^{k+1}} \sum_{l=0}^{M-1} l^{k}
$$


Remark 4. The sum of powers of natural numbers can be expressed in terms of Bernoulli numbers $B_{n}$ and can be easily tabulated [36,17]

$$
\begin{aligned}
\sum_{l=1}^{m} l^{k}= & \frac{m^{k+1}}{k+1}+\frac{m^{k}}{2}+\frac{1}{2}\left(\begin{array}{l}
k \\
1
\end{array}\right) B_{2} m^{k-1}+\frac{1}{4}\left(\begin{array}{l}
k \\
3
\end{array}\right) B_{4} m^{k-3} \\
& +\frac{1}{6}\left(\begin{array}{l}
k \\
5
\end{array}\right) B_{6} m^{k-5}+\ldots
\end{aligned}
$$

the last term containing either $m$ or $m^{2}$. It should be remarked that

$$
\frac{1}{m^{k+1}} \sum_{l=1}^{m} l^{k}=O\left(\frac{1}{k+1}\right), \quad \text { as } m \rightarrow \infty
$$

In this way, computing integrals of monomials $x^{j}$ multiplying $F(x / \varepsilon)$ over the reference domain $(0,1)$ reduces to computing the integrals of all monomials of degree $k$, with $0 \leq k \leq j$, multiplying $F(y)$ over the main period of $F(\cdot)$ and insert this in (15).

We comment on the development of the macroelement stiffness matrix for the case when the macro mesh $\mathcal{T}_{H}$ is aligned with the periodic lattice. If the shape functions of the micro space $\mathcal{M}^{\mu}$ are piecewise polynomials with respect to some unit-cell triangulation $\hat{\mathcal{T}}$, then the elemental stiffness matrices on the macro mesh can be evaluated exactly. For convenience, we consider only the 1-d case. For the calculation of the two-scale element stiffness matrices, the basic integrals

$$
\underline{\underline{K}}_{\mu}^{\gamma \delta \tau}=\left(\int_{\widehat{Q}} a(\hat{y}) \phi_{i}^{(\gamma)}(\hat{y}) \phi_{j}^{(\delta)}(\hat{y}) \hat{y}^{\tau} d \hat{y}\right)_{i, j=0, \ldots, \mu}
$$

are needed. For the case when $\mathcal{M}^{\mu}=S_{\mathrm{per}}^{\mu}(\widehat{Q}, \widehat{\mathcal{T}})$, i.e., the space of micro shape functions is the FE space corresponding to the FE discretization of the unit-cell, the macro stiffness and mass matrices can be developed from moments, i.e., integrals in the fast variable corresponding to discretization of the unit-cell problem with monomial weighted coefficients, combined with certain lattice summation formulas.

Let $K \in \mathcal{T}$ be a macro element of size $H$ with 'macroscopic' polynomial space $S^{p}(K)=\operatorname{Span}\left\{\nu_{I}^{[K]}\right\}_{I}$. The entries $\underline{\underline{A}}_{(I i)(J j)}^{\varepsilon,[K]}$ of the element stiffness matrix corresponding to $K$ are of the form

$$
\underline{\underline{A}}_{(I i)(J j)}^{\varepsilon,[K]}=\sum_{\gamma, \delta \leq 1} \sum_{\alpha=\alpha(p)} \sum_{\tau \leq \alpha}\left(\underline{\underline{K}}_{\mu}^{\gamma \delta \tau}\right)_{i j} \sum_{m=0}^{M-1} S_{\gamma \delta \alpha \tau}^{I J}(m, H, \varepsilon),
$$

with $M:=H / \varepsilon$ and with $\sum_{m=0}^{M-1} S_{\gamma \delta \alpha \tau}^{I J}(m, H, \varepsilon)$ being directly computable. The idea (see Lemma 3 ) is to compute sums of powers of natural numbers appearing in $\sum_{m=0}^{M} S_{\gamma \delta \alpha \tau}^{I J}(m, H, \varepsilon)$ in terms of Bernoulli numbers $B_{i}$. Thus, such sums can be computed with work independent of $M=H / \varepsilon$. More precisely, one exploits the fact that for $N \in \mathbb{N}, \sum_{k=1}^{N} k^{q}$ is given by

$$
\begin{aligned}
\sum_{k=1}^{N} k^{q}= & \frac{N^{q+1}}{q+1}+\frac{N^{q}}{2}+\frac{1}{2}\left(\begin{array}{l}
q \\
1
\end{array}\right) B_{2} N^{q-1}+\frac{1}{4}\left(\begin{array}{l}
q \\
3
\end{array}\right) B_{4} N^{q-3} \\
& +\frac{1}{6}\left(\begin{array}{l}
q \\
5
\end{array}\right) B_{6} N^{q-5}+\ldots,
\end{aligned}
$$


the last term containing either $N$ or $N^{2}$. It should be remarked that

$$
\frac{1}{N^{q+1}} \sum_{k=1}^{N} k^{q}=O\left(\frac{1}{q+1}\right), \quad \text { as } N \rightarrow \infty
$$

so that (17) could also be used as asymptotic expansion for small $\varepsilon=H / M$. The amount of work for computing the element stiffness matrix in the twoscale FEM is therefore independent of $\varepsilon$. If $n>1$, the same arguments apply if all indices are changed to suitable multi-indices.

In the remainder of this paper, we investigate the performance of the generalized FEM for homogenization problems. We start by investigating the regularity of the solutions $u^{\varepsilon}$ of (1) in dependence on $\varepsilon$ in the following section. Rather than using the classical asymptotic expansion technique (e.g. $[7,8,14])$, we use Fourier representations of the solution on the unbounded domain $\Omega_{\infty}^{\varepsilon}$ which are valid uniformly for all $\varepsilon>0$ to deduce uniform two-scale regularity of $u^{\varepsilon}$. Moreover, we will give finite dimensional approximations of $u^{\varepsilon}$ together with robust error estimates for the approximation error which are based on the Fourier representation. The finite dimensional approximations of $u^{\varepsilon}$ allow directly to deduce proper choices of $\mathcal{M}_{\varepsilon}^{\mu}$ in (14) so that corresponding error bounds are also valid for the two scale FEM.

\section{Regularity and Approximation of $u^{\varepsilon}$}

In this section we address problem (1) on unbounded domains, i.e., when $\Omega=$ $\mathbb{R}^{n}$. Based on a mixed variational formulation we show existence and uniqueness of the solution $u^{\varepsilon}$ in exponentially weighted Sobolev spaces $H_{-\nu}^{1}\left(\Omega_{\infty}^{\varepsilon}\right)$, $\nu \in\left(0, \nu_{0}\right)$, for general right hand sides $f$ in the dual space of $H_{\nu}^{1}\left(\Omega_{\infty}^{\varepsilon}\right)[31,26]$. We also represent $u^{\varepsilon}$ as a Fourier-Bochner integral with respect to the Banach spaces $H_{-\nu}^{1}\left(\Omega_{\infty}^{\varepsilon}\right)$. The starting point is the analysis of the Fourier-Bochner integral kernel introduced as the inverse Fourier symbol of the elliptic operator (1). In this symbol, scale separation occurs: it is the product of the standard Fourier waves $e^{i t \cdot x}$ and periodic functions $\phi(x / \varepsilon, \varepsilon, t)$ which encode the fast scale of the solution. More importantly, the functions $\phi(y, \varepsilon, t)$ arise as solutions of parameter dependent problems on the unit-cell $\widehat{Q}$ and are therefore amenable to FE-approximations. Next, we present the regularity properties of the integral kernel $e^{i t \cdot x} \phi(x / \varepsilon, \varepsilon, t)$ in dependence on the parameters $\varepsilon>0, t \in \mathbb{R}^{n}$. It turns out that the dependence on these parameters is analytic, even if the unit-cell problem admits only very low elliptic regularity. This analyticity is exploited in the construction of various finite-dimensional approximations of the solution as well as in proving robust error estimates for these approximations.

\subsection{Variational setting}

Let $\varepsilon>0$ be a length scale and let $\widehat{Q} \subset \widehat{Y}:=[-\pi, \pi]^{n}$ be a unit cell with Lipschitz boundary. We consider the homogenization problem on the unbounded domain

$$
\begin{gathered}
L^{\varepsilon}\left(\frac{x}{\varepsilon}, \partial_{x}\right) u^{\varepsilon}(x)=f(x) \text { in } \Omega_{\infty}^{\varepsilon} \\
n \cdot\left(a\left(\frac{x}{\varepsilon}\right) \nabla u^{\varepsilon}\right)=0 \text { on } \partial \Omega_{\infty}^{\varepsilon}
\end{gathered}
$$


in which the second order elliptic operator $L^{\varepsilon}\left(x / \varepsilon, \partial_{x}\right)$ is given by

$$
L^{\varepsilon}\left(\frac{x}{\varepsilon}, \partial_{x}\right) u(x):=-\nabla \cdot\left(a\left(\frac{x}{\varepsilon}\right) \nabla u\right)+a_{0}\left(\frac{x}{\varepsilon}\right) u,
$$

where $n$ is the unit outward normal to $\partial \Omega_{\infty}^{\varepsilon}, \varepsilon>0$ and $f \in L^{2}\left(\Omega_{\infty}^{\varepsilon}\right)$.

We considered homogenous Neumann boundary conditions on the interior cell boundaries, but emphasize that the case when $\widehat{Q}=\widehat{Y}$ or the case when homogeneous Dirichlet boundary conditions on the interior boundary $\partial \widehat{Q} \backslash \partial \widehat{Y}$ are prescribed can be treated completely analogously.

Then, by the Lax-Milgram Theorem [25], for every $f \in L^{2}\left(\Omega_{\infty}^{\varepsilon}\right)$ and for every $\varepsilon>0,(18)-(19)$ admits a unique weak solution $u^{\varepsilon} \in H^{1}\left(\Omega_{\infty}^{\varepsilon}\right)$, i.e.,

$$
B^{\varepsilon}\left(u^{\varepsilon}, v\right)=(f, v)_{L^{2}\left(\Omega_{\infty}^{\varepsilon}\right)} \quad \forall v \in H^{1}\left(\Omega_{\infty}^{\varepsilon}\right),
$$

where the bilinear form $B^{\varepsilon}(\cdot, \cdot)$ is given by

$$
B^{\varepsilon}(u, v):=\left(\nabla v, a\left(\frac{x}{\varepsilon}\right) \nabla u\right)_{L^{2}\left(\Omega_{\infty}^{\varepsilon}\right)}+\left(v, a_{0}\left(\frac{x}{\varepsilon}\right) u\right)_{L^{2}\left(\Omega_{\infty}^{\varepsilon}\right)}, \forall u, v \in H^{1}\left(\Omega_{\infty}^{\varepsilon}\right) .
$$

\subsection{Fourier representation of the solution}

Let $f=f(x)$ be independent of $\varepsilon$ and $f \in L^{2}\left(\mathbb{R}^{n}\right)$ (in particular, $f$ has to be defined everywhere on $\mathbb{R}^{n}$, not just on $\left.\Omega_{\infty}^{\varepsilon}\right)$. Then, the following Fourier inversion formula in $L^{2}\left(\mathbb{R}^{n}\right)$ holds

$$
f(x)=\frac{1}{(2 \pi)^{n / 2}} \int_{\mathbb{R}^{n}} \hat{f}(t) e^{i t \cdot x} d t
$$

where

$$
\hat{f}(t):=\frac{1}{(2 \pi)^{n / 2}} \int_{\mathbb{R}^{n}} e^{-i t \cdot x} f(x) d x \in L^{2}\left(\mathbb{R}^{n}\right)
$$

denotes the standard Fourier transform of $f$.

We might therefore think of $u^{\varepsilon}(x)$ as a superposition of elementary solutions $\hat{f}(t) \psi^{\varepsilon}(x, t)$ of (18)-(19) with right hand side $\hat{f}(t) e^{i t \cdot x}$, where the kernel $\psi^{\varepsilon}(x, t)$ satisfies

$$
\begin{gathered}
L^{\varepsilon}\left(x / \varepsilon, \partial_{x}\right) \psi^{\varepsilon}(x, t)=e^{i t \cdot x} \quad \text { in } \Omega_{\infty}^{\varepsilon}, \\
n \cdot a\left(\frac{x}{\varepsilon}\right) \nabla_{x} \psi^{\varepsilon}(x, t)=0 \quad \text { on } \quad \partial \Omega_{\infty}^{\varepsilon} .
\end{gathered}
$$

To this end, we have to give a rigorous mathematical meaning to the integral

$$
\int_{\mathbb{R}^{n}} \hat{f}(t) \psi^{\varepsilon}(x, t) d t
$$

Since $f(x)=e^{i t \cdot x}$ in $(21)$ is not in $L^{2}\left(\Omega_{\infty}^{\varepsilon}\right),(21)$ has no solution in $H^{1}\left(\Omega_{\infty}^{\varepsilon}\right)$ in the sense of (20). We have therefore to weaken the notion of solution of (18)-(19) and to allow for input data such as $f(x)=e^{i t \cdot x}$.

To characterize precisely this notion of solution of (18)-(19) in $\Omega_{\infty}^{\varepsilon}$, we introduce weighted Sobolev spaces $H_{\nu}^{j}\left(\Omega_{\infty}^{\varepsilon}\right)$ of complexed-valued functions with exponential weights depending on a real parameter $\nu$. 
Definition 5. For $j=0,1$ and for any $\nu \in \mathbb{R}$ the weighted Sobolev spaces $H_{\nu}^{j}\left(\Omega_{\infty}^{\varepsilon}\right)$ equipped with the norm $\|\cdot\|_{j, \nu}$ are defined to be

$$
H_{\nu}^{j}\left(\Omega_{\infty}^{\varepsilon}\right)=\overline{\left.C_{0}^{\infty}\left(\mathbb{R}^{n} ; \mathbb{C}\right)\right|_{\Omega_{\infty}^{\varepsilon}}}\|\cdot\|_{j, \nu},
$$

where

$\|u\|_{j, \nu}^{2}=\int_{\Omega_{\infty}^{\varepsilon}}\left(\sum_{|\alpha| \leq j}\left|D_{x}^{\alpha} u\right|^{2}\right) e^{2 \nu\|x\|} d x \quad\left(D_{x}^{\alpha} u=\partial_{x_{1}}^{\alpha_{1}} \ldots \partial_{x_{n}}^{\alpha_{n}} u, \forall \alpha \in \mathbb{N}_{0}^{n}\right)$.

Note that for $\nu>0$ holds $H_{\nu}^{1} \subset H_{0}^{1}=H^{1} \subset H_{-\nu}^{1}$. The variational solution $u^{\varepsilon} \in H^{1}\left(\Omega_{\infty}^{\varepsilon}\right)$ of (20) can also be viewed as an element of the exponentially weighted Sobolev spaces $H_{-\nu}^{1}\left(\Omega_{\infty}^{\varepsilon}\right)$ for some $\nu>0$. Moreover, it has the following representation: for $x \in \Omega_{\infty}^{\varepsilon}$, there holds

$$
u^{\varepsilon}(x)=\frac{1}{(2 \pi)^{n / 2}} \int_{\mathbb{R}^{n}}^{(B)} \hat{f}(t) \psi^{\varepsilon}(x, t) d t .
$$

Remark 6 . Here and in the following we write $\int^{(B)}$ to designate a Bochner integral of $H_{-}^{1}$-valued functions.

To render (21), (24) meaningful, we must generalize (20) for right hand sides $f(x)$ which are not decaying at $\infty$. To do so, we extend $B^{\varepsilon}(.,$.$) to a sesquilin-$ ear form $\Psi(\varepsilon)[\cdot, \cdot]: H_{-\nu}^{1}\left(\Omega_{\infty}^{\varepsilon}\right) \times H_{\nu}^{1}\left(\Omega_{\infty}^{\varepsilon}\right) \rightarrow \mathbb{C}:$

$$
\begin{aligned}
& \Psi(\varepsilon)[u, v]= \\
& \int_{\Omega_{\infty}^{\varepsilon}}\left\{\sum_{p, q=1}^{n} a_{p q}\left(\frac{x}{\varepsilon}\right) \frac{\partial u}{\partial x_{p}}(x) \overline{\frac{\partial v}{\partial x_{q}}(x)}+a_{0}\left(\frac{x}{\varepsilon}\right) u(x) \overline{v(x)}\right\} d x .
\end{aligned}
$$

For all $\varepsilon>0$ and for $\nu>0$ sufficiently small, $\Psi(\varepsilon)$ is bounded and 'coercive' with respect to $H_{-\nu}^{1}\left(\Omega_{\infty}^{\varepsilon}\right) \times H_{\nu}^{1}\left(\Omega_{\infty}^{\varepsilon}\right)$, in the sense that the inf-sup stability condition holds (note that for $\nu=0, \Psi(\varepsilon)$ coincides with $B^{\varepsilon}$ in (20)). More precisely, there holds $[31,32,26]$ :

Proposition 7. There exist positive constants $\nu_{0}, C$ and $\gamma$ such that for all $\nu \in\left(0, \nu_{0}\right)$ and all $\varepsilon>0$

$$
\begin{aligned}
& \text { 1. }|\Psi(\varepsilon)[u, v]| \leq C\|u\|_{1,-\nu}\|v\|_{1, \nu} \text {, } \\
& \text { 2. } \inf _{\|u\|_{1,-\nu}=1} \sup _{\|v\|_{1, \nu}=1}|\Psi(\varepsilon)[u, v]| \geq \gamma>0 \\
& \text { 3. } \sup _{u \in H_{-\nu}^{1}\left(\Omega_{\infty}^{\varepsilon}\right)}|\Psi(\varepsilon)[u, v]|>0 \text { for all } v \in H_{\nu}^{1}\left(\Omega_{\infty}^{\varepsilon}\right) \text { and } v \neq 0 .
\end{aligned}
$$

The continuity of the sesquilinear form $\Psi(\varepsilon)$ stated in 1 . is obvious. The inf-sup condition 2. and the injectivity property 3. can be verified in the following way: based on the coercivity of the bilinear form $B^{\varepsilon}$ for the case $\nu=0$ a perturbation argument can be employed to prove the existence of a positive $\nu_{0}>0$ such that 2 . and 3 . hold. We emphasize that $\nu_{0}$ is independent of $\varepsilon$ and depends only on the upper and lower bounds of the matrix $a$ and of the zero order coefficient $a_{0}$. Proposition 7 implies immediately the existence of $\psi^{\varepsilon}(x, t)$ : 
Proposition 8. The properties 1, 2 and 3 of $\Psi(\varepsilon)$ imply that the variational problem

Given $f \in\left(H_{\nu}^{1}\left(\Omega_{\infty}^{\varepsilon}\right)\right)^{*}$, find

$u \in H_{-\nu}^{1}\left(\Omega_{\infty}^{\varepsilon}\right): \Psi(\varepsilon)[u, v]=\langle f, v\rangle_{\left(H_{\nu}^{1}\left(\Omega_{\infty}^{\varepsilon}\right)\right)^{*} \times H_{\nu}^{1}\left(\Omega_{\infty}^{\varepsilon}\right)}, \quad \forall v \in H_{\nu}^{1}\left(\Omega_{\infty}^{\varepsilon}\right)$,

admits a unique weak solution $u \in H_{-\nu}^{1}\left(\Omega_{\infty}^{\varepsilon}\right)$ and the a-priori estimate

$$
\|u\|_{H_{-\nu}^{1}\left(\Omega_{\infty}^{\varepsilon}\right)} \leq(1 / \gamma)\|f\|_{\left(H_{\nu}^{1}\left(\Omega_{\infty}^{\varepsilon}\right)\right)^{*}}
$$

holds.

Since $e^{i t \cdot x} \in\left(H_{\nu}^{1}\left(\Omega_{\infty}^{\varepsilon}\right)\right)^{*}$, this result implies the existence of a solution $\psi^{\varepsilon}(x, t)$ of (21). The representation (24), however, is yet of limited use for applications since the kernel $\psi^{\varepsilon}(x, t)$ requires the solution of an unbounded domain problem and is not explicitly available. However, in the problem (18), (19) holds scale-separation which is reflected in the structure of the kernel $\psi^{\varepsilon}(x, t)$, as will now be explained.

Let $\phi(\cdot, \varepsilon, t)$ be the $2 \pi$-periodic weak solution of the so-called unit-cell problem

$$
\begin{aligned}
\mathcal{L}\left(\varepsilon, t ; y, \partial_{y}\right) \phi & :=e^{-i \varepsilon t 3 \cdot y} L^{\varepsilon}\left(y, \varepsilon^{-1} \partial_{y}\right) e^{i \varepsilon t \cdot y} \phi \\
& =-e^{-i \varepsilon t \cdot y} \sum_{p, q=1}^{n} \varepsilon^{-2} \frac{\partial}{\partial y_{p}}\left(a_{p q}(y) \frac{\partial}{\partial y_{q}}\left(\phi(y, \varepsilon, t) e^{i t \cdot \varepsilon y}\right)\right) \\
& +a_{0}(y) \phi(y, \varepsilon, t)=1, \quad \text { in } \widehat{Q} \\
n \cdot a(y)(i t \varepsilon & \left.+\nabla_{y}\right) \phi=0 \text { on } \widehat{\Gamma}_{N} .
\end{aligned}
$$

We denoted here by $\widehat{\Gamma}_{N}$ the Neumann boundary $\widehat{\Gamma}_{N}:=\partial \widehat{Q} \backslash \widehat{\Gamma}_{\text {per }}$, where $\widehat{\Gamma}_{\text {per }}=\partial \widehat{Q} \cap \partial[-\pi, \pi]^{n}$.

We will refer to $x \in \mathbb{R}^{n}$ as the slow variable and $y=x / \varepsilon$ will denote the fast variable of the problem.

The Sobolev spaces of periodic functions on $\Omega_{\infty}$ for which $\widehat{Y}$ is the fundamental period will be denoted by $H_{\mathrm{per}}^{j}(\widehat{Q})$, and are defined to be the completion with respect to the standard Sobolev norm $\|\cdot\|_{j}$ of the complex-valued, $C^{\infty}$ functions on $\Omega_{\infty}$ that are $2 \pi$-periodic in each coordinate. Next, we define

$$
\psi(y, \varepsilon, t):=\phi(y, \varepsilon, t) e^{i t \cdot \varepsilon y}, \quad y \in \Omega_{\infty} .
$$

The function $\psi$ is the generalized fundamental solution of $L^{\varepsilon}$ (resp. the twoscale Fourier symbol of $\left(L^{\varepsilon}\right)^{-1}$ on $\left.\Omega_{\infty}^{\varepsilon}\right)$. The basic tool for the proof of the Fourier-Bochner integral representation in (24) is given in the following proposition:

Proposition 9. For each $\varepsilon>0, t \in \mathbb{R}^{n}$ and $\nu \in\left(0, \nu_{0}\right)$, the kernel $\psi$ defined in (28) is the unique weak solution in $H_{-\nu}^{1}\left(\Omega_{\infty}^{\varepsilon}\right)$ of

$$
\Psi(\varepsilon)\left[\psi\left(\frac{\dot{-}}{\varepsilon}, \varepsilon, t\right), v\right]=\left\langle e^{i t \cdot x}, v\right\rangle_{\left(H_{\nu}^{1}\right)^{*} \times H_{\nu}^{1}}, \quad \forall v \in H_{\nu}^{1}\left(\Omega_{\infty}^{\varepsilon}\right) .
$$

In particular therefore $\psi^{\varepsilon}(x, t)=\psi(x / \varepsilon, \varepsilon, t)$ in $H_{-\nu}^{1}\left(\mathbb{R}^{n}\right)$ and

$$
\left\|\psi\left(\frac{x}{\varepsilon}, \varepsilon, t\right)\right\|_{1,-\nu} \leq \frac{1}{\gamma \nu^{n / 2}} .
$$


We state now the Fourier-Bochner integral respresentation formula (24) in weighted Sobolev spaces for the solution $u^{\varepsilon}(x)$ of (18) on $\Omega_{\infty}^{\varepsilon}$. The proof can be found in [31].

Theorem 10. Let $f(x) \in L^{2}\left(\mathbb{R}^{n}\right), \nu \in\left(0, \nu_{0}\right)$ and $\varepsilon>0$. Further let $u^{\varepsilon}(x) \in$ $H^{1}\left(\Omega_{\infty}^{\varepsilon}\right)$ be the solution of (18) on $\Omega_{\infty}^{\varepsilon}$. Then, as an element of the weighted Sobolev space $H_{-\nu}^{1}\left(\Omega_{\infty}^{\varepsilon}\right)$, $u^{\varepsilon}$ admits the Bochner integral representation (24).

We will show how the kernels $\phi(y, \varepsilon, t), \psi(y, \varepsilon, t)$ can be used to design FE subspaces of $(1),(2)$ which encode the microstructure and coefficient regularity. A crucial role for obtaining error estimates will be played by the kernels' analyticity with respect to $t$. As a byproduct of the analysis, we obtain specific choices of the micro-subspace $\mathcal{M}_{\varepsilon}^{\mu}$ in the generalized FE-space (14).

\subsection{Regularity of the kernels $\psi, \phi$}

The kernel $\phi(\cdot, \varepsilon, t)$ can be continued analytically with respect to $(\varepsilon, t)$ in a complex neighborhood $\widehat{G} \subset \mathbb{C}^{n+1}$ of $\mathbb{R}^{n+1}$, with values in $H_{\text {per }}^{1}(\widehat{Q})$. More precisely, for every fixed $\varepsilon>0$, the mappings

$$
\mathbb{R}^{n} \ni t \rightarrow \phi\left(\frac{\dot{\varepsilon}}{\varepsilon}, \varepsilon, t\right), \psi\left(\frac{\dot{ }}{\varepsilon}, \varepsilon, t\right) \in H_{-\nu}^{1}\left(\Omega_{\infty}^{\varepsilon}\right)
$$

can be continued analytically with respect to $t$ in a strip neighbourhood of $\mathbb{R}^{n}$, and the width of the strip is independent of $\varepsilon$.

Let $\nu \in\left(0, \nu_{0}\right), \nu_{n}=\nu /(2 \sqrt{n})$ and denote by $\mathcal{D}_{\nu_{n}}$ the strip region of width $\nu_{n}$

$$
\mathcal{D}_{\nu_{n}}:=\left\{t \in \mathbb{C}^{n}: \max _{j=1, \ldots, n}\left|\operatorname{Im} t_{j}\right|<\nu_{n}\right\}
$$

It can be verified that the mapping

$$
\mathcal{D}_{\nu_{n}} \ni t \rightarrow G(t):=e^{i t \cdot x} \in\left(H_{\nu}^{1}\left(\Omega_{\infty}^{\varepsilon}\right)\right)^{*}
$$

is holomorphic in $\mathcal{D}_{\nu_{n}}$ with values in the Banach space $\left(H_{\nu}^{1}\left(\Omega_{\infty}^{\varepsilon}\right)\right)^{*}$. Moreover, $G_{k}(t):=(i x)^{k} e^{i t \cdot x} \in\left(H_{\nu}^{1}\left(\Omega_{\infty}^{\varepsilon}\right)\right)^{*}$ is the $k \in \mathbb{N}^{n}$-th partial derivative with respect to $t$ of the $\left(H_{\nu}^{1}\left(\Omega_{\infty}^{\varepsilon}\right)\right)^{*}$-valued mapping $G(t)$ and its norm has the following bound

$$
\forall t \in \mathcal{D}_{\nu_{n}}: \quad\left\|G_{k}(t)\right\|_{\left(H_{\nu}^{1}\left(\Omega_{\infty}^{\varepsilon}\right)\right)^{*}} \leq C_{\nu, n} \frac{2^{|k|} k !}{\nu^{|k|}} n^{|k| / 2}, \quad k \in \mathbb{N}^{n},
$$

where $k !:=k_{1} ! \ldots k_{n}$ !. For fixed $t \in \mathcal{D}_{\nu_{n}}$ and $k \in \mathbb{N}^{n}$, let $\psi_{k}^{\varepsilon}(t)$ be the weak solution in $H_{-\nu}^{1}\left(\Omega_{\infty}^{\varepsilon}\right)$ of the following variational problem:

$$
\begin{aligned}
& \psi_{k}^{\varepsilon} \in H_{-\nu}^{1}\left(\Omega_{\infty}^{\varepsilon}\right): \Psi(\varepsilon)\left[\psi_{k}^{\varepsilon}(t), v\right]= \\
& \left\langle G_{k}(t), v\right\rangle_{\left(H_{\nu}^{1}\left(\Omega_{\infty}^{\varepsilon}\right)\right)^{*} \times H_{\nu}^{1}\left(\Omega_{\infty}^{\varepsilon}\right)}, \quad \forall v \in H_{\nu}^{1}\left(\Omega_{\infty}^{\varepsilon}\right) .
\end{aligned}
$$

By Proposition 8, $\psi_{k}^{\varepsilon}$ exists and is unique. It has the following properties (see [26] for a proof):

1) The mapping $\mathcal{D}_{\nu_{n}} \ni t \rightarrow \psi(\cdot / \varepsilon, \varepsilon, t) \in H_{-\nu}^{1}\left(\Omega_{\infty}^{\varepsilon}\right)$ is holomorphic in $\mathcal{D}_{\nu_{n}}$ with values in the Banach space $H_{-\nu}^{1}\left(\Omega_{\infty}^{\varepsilon}\right)$. Moreover, $\psi_{k}^{\varepsilon}(t)$ defined by (33) is given by

$$
\psi_{k}^{\varepsilon}(t)=D_{t}^{k} \psi\left(\frac{\dot{\sigma}}{\varepsilon}, \varepsilon, t\right)=\partial_{t_{1}}^{k_{1}} \ldots \partial_{t_{n}}^{k_{n}} \psi(\dot{\bar{\varepsilon}}, \varepsilon, t)
$$


and the $\|\cdot\|_{1,-\nu}$-norm of $\psi_{k}^{\varepsilon}(t)$ is uniformly bounded with respect to $t \in \mathcal{D}_{\nu_{n}}$ by

$$
\left\|\psi_{k}^{\varepsilon}(t)\right\|_{1,-\nu} \leq C_{\nu, n} \frac{2^{|k|} k !}{\gamma \nu^{|k|}} n^{|k| / 2}
$$

2) For a given $\nu \in\left(0, \nu_{0}\right)$ the mapping $\mathcal{D}_{\nu_{n}} \ni t \rightarrow \phi(\cdot / \varepsilon, \varepsilon, t) \in H_{-2 \nu}^{1}\left(\Omega_{\infty}^{\varepsilon}\right)$ is a holomorphic function of $t \in \mathcal{D}_{\nu_{n}}$ with values in the Banach space $H_{-2 \nu}^{1}\left(\Omega_{\infty}^{\varepsilon}\right)$. Moreover,

$$
\left\|D_{t}^{k} \phi\left(\frac{\dot{-}}{\varepsilon}, \varepsilon, t\right)\right\|_{H_{-2 \nu}^{1}\left(\Omega_{\infty}^{\varepsilon}\right)} \leq C_{\nu, n, \gamma}(1+\|t\|) \frac{(k+\mathbf{1}) !}{(\nu / 2)^{|k|}} n^{|k| / 2}, \forall t \in \mathcal{D}_{d},
$$

where $\mathbf{1}:=(1,1, \ldots, 1)^{\top} \in \mathbb{R}^{n}$.

\subsection{Approximation of $\boldsymbol{u}^{\varepsilon}$}

The analyticity of the kernel $\psi(y, \varepsilon, t)$ with respect to $\varepsilon$ can be used to obtain from (24) asymptotic expansions with respect to $\varepsilon$ which essentially reproduce the classical asymptotic homogenizations of (1), (2). Here, we derive local approximation spaces $\mathcal{V}_{\varepsilon}^{N}$ from approximations of $u^{\varepsilon}$ based on a Poisson summation formula applied to the Bochner representation (24). This spectral approach gives robust, exponentially convergent approximations of $u^{\varepsilon}$ under certain analyticity properties of the data.

\section{Sets of analytic data}

Assume that $X$ is a Banach space and let $\mathcal{D}_{d}$ denote the strip region of width $d$ given by

$$
\mathcal{D}_{d}=\left\{z \in \mathbb{C}^{n}:|\operatorname{Im}(z)| \leq d\right\}
$$

Then we define

$$
\begin{aligned}
& \mathbf{H}^{p}\left(\mathcal{D}_{d} ; X\right)= \\
& \left\{g: \mathcal{D}_{d} \rightarrow X \mid g \text { is analytic in } \mathcal{D}_{d} \text { and } \mathbf{N}_{p}\left(g, \mathcal{D}_{d} ; X\right)<\infty\right\}
\end{aligned}
$$

where

$$
\mathbf{N}_{p}\left(g, \mathcal{D}_{d} ; X\right)=\left\{\begin{array}{cl}
\lim _{\delta \rightarrow 0^{+}}\left(\int_{\partial \mathcal{D}_{d}(\delta)}\|g(z)\|_{X}^{p}|d z|\right)^{1 / p}, & \text { if } 1 \leq p<\infty \\
\lim _{\delta \rightarrow 0^{+}} \sup _{z \in \mathcal{D}_{d}(\delta)}\|g(z)\|_{X} & , \quad \text { if } p=\infty,
\end{array}\right.
$$

and for $0<\delta<1, \mathcal{D}_{d}(\delta)$ is defined by

$$
\mathcal{D}_{d}(\delta)=\left\{z \in \mathbb{C}^{n}:|\operatorname{Re}(z)|<1 / \delta,|\operatorname{Im}(z)|<d(1-\delta)\right\}
$$

Definition 11. We say, a function $f$ fulfils the 'usual assumptions', if $f \in$ $L^{2}\left(\mathbb{R}^{n}\right)$, and its Fourier transformation can be extended to a holomorphic function $\hat{f}$ in the strip $\mathcal{D}_{d}$, with $d=\nu_{n}$ as in (30), that satisfies the following growth condition:

$$
|\hat{f}(z)| \leq C(f) e^{-\alpha\|z\|}, \quad \forall z \in \mathcal{D}_{d}
$$

for some positive constants $C(f), \alpha>0$. 
Remark 12. A typical example of functions that satisfy the 'usual assumptions' is that of functions arising through convolution with a Gauss kernel. Let $f \in L^{2}\left(\mathbb{R}^{n}\right)$ be compactly supported. Without loss of generality we may assume that $\operatorname{supp} f \subset\{x:\|x\| \leq 1\}$. Further let $\delta>0$ and denote by $\rho_{\delta}$ the following Dirac sequence

$$
\rho_{\delta}(y):=\delta^{-n / 2} e^{-\|y\|^{2} / \delta}, \quad y \in \mathbb{R}^{n} .
$$

Define $f_{\delta}:=f * \rho_{\delta}$, i.e.,

$$
f_{\delta}(x)=\delta^{-n / 2} \int_{\mathbb{R}^{n}} f(y) e^{-\|x-y\|^{2} / \delta} d y, \quad x \in \mathbb{R}^{n} .
$$

Then, $f_{\delta}$ defined by (39) is an analytic, exponentially decaying function that satisfies the 'usual assumptions'. Indeed, the Fourier transform of $f_{\delta}$ is given by the product of the Fourier transforms of $f$ and $\rho_{\delta}$

$$
\hat{f}_{\delta}(\xi)=\hat{f}(\xi) \hat{\rho}_{\delta}(\xi)=e^{-\delta\|\xi\|^{2} / 4} \hat{f}(\xi)
$$

which is an analytic function that decays exponentially in a complex strip neighborhood of $\mathbb{R}^{n}$.

Under the 'usual assumptions' on $f$ the mapping

$$
\mathcal{D}_{d} \ni t \rightarrow g(t, \cdot)=g_{\varepsilon}(t, \cdot):=\frac{1}{(2 \pi)^{n / 2}} \hat{f}(t) \phi\left(\frac{\dot{\varepsilon}}{\varepsilon}, \varepsilon, t\right) \in H_{-2 \nu}^{1}\left(\Omega_{\infty}^{\varepsilon}\right)
$$

is in $\mathbf{H}^{p}\left(\mathcal{D}_{d} ; H_{-2 \nu}^{1}\left(\Omega_{\infty}^{\varepsilon}\right)\right)$. Moreover, there exists a $C(\gamma, \nu, n)$ such that $g_{\varepsilon}(t, \cdot)$ satisfies the growth condition:

$$
\left\|g_{\varepsilon}(t, \cdot)\right\|_{1,-2 \nu} \leq C(\gamma, \nu, n) C(f)\left(1+\frac{1}{\alpha}\right) e^{-\frac{\alpha}{2}\|t\|}, \quad \forall t \in \mathcal{D}_{d},
$$

where $\alpha$ and $C(f)$ are as in (38).

\section{Trapezoidal approximation of (24)}

We shall now derive finite dimensional approximations by truncating a generalized Poisson summation formula applied to the Bochner representation

$$
\begin{aligned}
u^{\varepsilon}(x) & =\frac{1}{(2 \pi)^{n / 2}} \int_{\mathbb{R}^{n}}^{(B)} \hat{f}(t) \psi(x / \varepsilon, \varepsilon, t) d t \\
& =\frac{1}{(2 \pi)^{n / 2}} \int_{\mathbb{R}^{n}}^{(B)} \hat{f}(t) e^{i t \cdot x} \phi\left(\frac{x}{\varepsilon}, \varepsilon, t\right) d t .
\end{aligned}
$$

Let $L \geq 1$ and assume in what follows that $\pi / h \geq 2 L$, i.e., $h \leq \pi /(2 L)$. We define

$$
\mathbb{Z}_{(N)}^{n}:=\left\{k \in \mathbb{Z}^{n}:\left|k_{p}\right| \leq N, \forall p=1, \cdots, n\right\}
$$

and the trapezoidal sum approximations of the Bochner integral (42) is

$$
\begin{aligned}
u_{N, h}^{\varepsilon}(x) & =\mathbf{1}_{\left[-\frac{\pi}{h}, \frac{\pi}{h}\right]^{n}}(x) \frac{1}{(2 \pi)^{n / 2}} h^{n} \sum_{k \in \mathbb{Z}_{(N)}^{n}} \phi\left(\frac{x}{\varepsilon}, \varepsilon, k h\right) \hat{f}(k h) e^{i k h \cdot x} \\
& =\mathbf{1}_{\left[-\frac{\pi}{h}, \frac{\pi}{h}\right]^{n}}(x) \frac{1}{(2 \pi)^{n / 2}} h^{n} \sum_{k \in \mathbb{Z}_{(N)}^{n}} \psi\left(\frac{x}{\varepsilon}, \varepsilon, k h\right) \hat{f}(k h) .
\end{aligned}
$$

Then, $u^{\varepsilon}$ in the integral $(24)$ can be split as

$$
u^{\varepsilon}(\cdot)=u_{N, h}^{\varepsilon}(\cdot)+\delta_{N}(f, h)(\cdot) .
$$


Remark 13. Since we have real-valued input data we have a real valued solution $u^{\varepsilon}$. The same holds for the finite approximation $u_{N, h}^{\varepsilon}$. Indeed, since

$$
\hat{f}(-\xi)=\overline{\hat{f}(\xi)}, \quad \psi\left(\frac{\dot{\varepsilon}}{\varepsilon}, \varepsilon,-\xi\right)=\overline{\psi\left(\frac{\dot{q}}{\varepsilon}, \varepsilon, \xi\right)},
$$

it follows that

$$
\begin{aligned}
& u_{N, h}^{\varepsilon}(x)= \\
& \mathbf{1}_{\left[-\frac{\pi}{h}, \frac{\pi}{h}\right]^{n}}(x) \frac{1}{(2 \pi)^{n / 2}} h^{n} \psi\left(\frac{x}{\varepsilon}, \varepsilon, 0\right) \hat{f}(0)+2 \mathbf{1}_{\left[-\frac{\pi}{h}, \frac{\pi}{h}\right]^{n}}(x) \frac{1}{(2 \pi)^{n / 2}} h^{n} \\
& \times \sum_{k \in \mathbb{Z}_{(N)}^{n} \backslash\{0\}, k_{1} \geq 0}\left\{\operatorname{Re} \psi\left(\frac{x}{\varepsilon}, \varepsilon, k h\right) \operatorname{Re} \hat{f}(k h)-\operatorname{Im} \psi\left(\frac{x}{\varepsilon}, \varepsilon, k h\right) \operatorname{Im} \hat{f}(k h)\right\},
\end{aligned}
$$

which is real valued.

We next present two types of robust (in $\varepsilon$ ) convergence estimates which are based on different assumptions on the data $f$.

\section{Exponential convergence}

Under the 'usual assumptions' on the data $f$, the sum (43) approximates $u^{\varepsilon}$ in $(24)$ at a robust exponential rate, i.e., independent of $\varepsilon$, (see $[26,29]$ ).

Proposition 14. Let $f$ satisfy the 'usual assumptions' in Definition 11 with some $\alpha, d>0$ and let $L>0$ be arbitrary. Define

$$
h=\left(\frac{\pi d}{\alpha N}\right)^{1 / 2}
$$

and assume $N \geq 4 d L^{2} /(\alpha \pi)$, i.e., $\pi / h \geq 2 L$. Then, the error $\delta_{N}(f, h)(\cdot)=$ $u^{\varepsilon}(\cdot)-u_{N, h}^{\varepsilon}(\cdot)$, with $u_{N, h}^{\varepsilon}(\cdot)$ as in (43), decays exponentially with respect to $N$ and uniformly with respect to $\varepsilon$ in the $\|\cdot\|_{0,-2 \nu},\|\cdot\|_{H_{-2 \nu}^{1}\left((-L, L)^{n}\right)^{-n o r m s:}}$

$$
\begin{aligned}
& \left\|\delta_{N}(f, h)(\cdot)\right\|_{0,-2 \nu}+\left\|\delta_{N}(f, h)(\cdot)\right\|_{H_{-2 \nu}^{1}\left((-L, L)^{n}\right)} \\
& \quad \leq C(\gamma, \nu, n) C(f)\left(1+\frac{1}{\alpha}\right)^{2} \frac{1}{\alpha^{n}} e^{-(\pi \alpha d N / n)^{1 / 2}} .
\end{aligned}
$$

The constants $C(\gamma, \nu, n), C(f)$ are independent of $\varepsilon, N, L$.

As a corollary, the following approximation result holds

Corollary 15. Assume that $f$ satisfies the 'usual assumptions' and that

$$
h=\left(\frac{\pi d}{\alpha N}\right)^{1 / 2}, \quad N \geq \frac{4 d L^{2}}{\alpha \pi} .
$$

Define the finite-dimensional subspace

$$
\mathcal{V}_{\varepsilon}^{N}:=\operatorname{Span}\left\{\operatorname{Re} \psi\left(\frac{\dot{\varepsilon}}{\varepsilon}, \varepsilon, k h\right), \operatorname{Im} \psi\left(\frac{\dot{\cdot}}{\varepsilon}, \varepsilon, k h\right): k \in \mathbb{Z}_{(N)}^{n}\right\} .
$$

Then

$$
\begin{gathered}
\inf _{v \in \mathcal{V}_{\varepsilon}^{N}}\left\|u^{\varepsilon}-v\right\|_{H_{-2 \nu}^{1}\left((-L, L)^{n}\right)}+\inf _{v \in \mathcal{V}_{\varepsilon}^{N}}\left\|u^{\varepsilon}-v\right\|_{0,-2 \nu} \\
\leq C(\gamma, \nu, n) C(f)\left(1+\frac{1}{\alpha}\right)^{2} \frac{1}{\alpha^{n}} e^{-(\pi \alpha d N / n)^{1 / 2}},
\end{gathered}
$$

where $C(f)$ and $\alpha=\alpha(f)$ are those from Definition 11, in particular independent of $\varepsilon, N$. 


\section{Spectral convergence}

The usual assumptions on the data $f$ entail essentially boundedness of all derivatives. Now we assume that $f(x) \in H^{s}\left(\mathbb{R}^{n}\right)$ in (18) has finite regularity $(s>n / 2)$ and is compactly supported, i.e., $f \in H_{\text {comp }}^{s}\left(\mathbb{R}^{n}\right)$. Then for any $\varepsilon>0$ the solution $u^{\varepsilon}(x)$ of (24) can be approximated by

$$
u_{N}^{\varepsilon} \in \mathcal{V}_{\varepsilon}^{N}=\operatorname{Span}\{\operatorname{Re} \psi(\cdot / \varepsilon, \varepsilon, k h), \operatorname{Im} \psi(\cdot / \varepsilon, \varepsilon, k h)\}_{k \in \mathbb{Z}_{(N)}^{n}}
$$

with respect to $\|\cdot\|_{1,-\nu}$ at a robust algebraic convergence rate.

Proposition 16. Assume that $f$ in (18) is $H^{s}\left(\mathbb{R}^{n}\right)$ with $s>n / 2$ and supp $f \subset$ $\Omega_{M}:=(-M, M)^{n}$, with $M>0$. Let $d:=\min \left\{1 / M, \nu_{n}\right\}, \nu_{n}=1 /(2 \sqrt{n})$, and $N \geq 1$. Then, for any $\varepsilon>0$ and for all $N$

$$
\inf _{v \in \mathcal{V}_{\varepsilon}^{N}}\left\|u^{\varepsilon}-v\right\|_{1,-\nu} \leq C_{\nu, s, n} N^{-(s-n / 2) / 2}\|f\|_{H^{s}\left(\mathbb{R}^{n}\right)},
$$

where $C_{\nu, s, n}>0$ is independent of $\varepsilon, N$, and $M$.

\section{Approximation Properties on the Bounded Domain}

On the unbounded domain, the spaces (47) have robust (with respect to $\varepsilon)$ approximation properties. Thus we obtained rates of convergence that were independent of the regularity of the coefficients and of the unit-cell problem (Proposition 14, Corollary 15). We base a generalized FEM in $\Omega^{\varepsilon}$ on the spaces $\mathcal{V}_{\varepsilon}^{N}$ from the unbounded domain. However, the spaces $\mathcal{V}_{\varepsilon}^{N}$ are not $H_{\Gamma_{D}^{\varepsilon}}^{1}\left(\Omega^{\varepsilon}\right)$-conforming, i.e., $\mathcal{V}_{\varepsilon}^{N} \not \subset H_{\Gamma_{D}^{\varepsilon}}^{1}\left(\Omega^{\varepsilon}\right)$ in general. Thus, $\mathcal{V}_{\varepsilon}^{N}$ cannot be used as FE-spaces directly and have to be modified. Our approch for enforcing $H_{\Gamma_{D}^{\varepsilon}}^{1}\left(\Omega^{\varepsilon}\right)$-conformity is to use a standard FEM in a neighborhood of the boundary and the spaces $\mathcal{V}_{\varepsilon}^{N}$ in the interior of the domain.

\subsection{Density of the approximation spaces}

A minimal requirement for the generalized FEM (7) is that $\cup_{N} V_{N}$ be dense (in the $H^{1}\left(\Omega^{\varepsilon}\right)$-norm) in the set of all solutions. Since we will base our spaces $V_{N}$ on the spaces $\mathcal{V}_{\varepsilon}^{N}$ in (47), we first ascertain in Proposition 17 that $\cup_{N} \mathcal{V}_{\varepsilon}^{N}$ is dense in the set of solutions of (1), (2) with boundary conditions as in (11).

Proposition 17. Let $f \in L^{2}(\Omega)$ and let $u^{\varepsilon} \in H_{\Gamma_{D}^{\varepsilon}}^{1}\left(\Omega^{\varepsilon}\right)$ be the solution of (1), (11). Then, for all bounded Lipschitz domains $\Omega_{0} \subset \subset \Omega$, with $\Omega_{0}^{\varepsilon}:=$ $\Omega_{0} \cap \Omega_{\infty}^{\varepsilon}$

$$
\lim _{N \rightarrow \infty} \inf _{v \in \cup_{\mu=1}^{N}} \mathcal{V}_{\varepsilon}^{\mu}\left\|u^{\varepsilon}-v\right\|_{H^{1}\left(\Omega_{0}^{\varepsilon}\right)}=0
$$

i.e., $\left.\cup_{N>0} \mathcal{V}_{\varepsilon}^{N}\right|_{\Omega}$ is dense with respect to the $H^{1}\left(\Omega_{0}^{\varepsilon}\right)$-topology in the space of solutions to (5) corresponding to right-hand sides $f \in L^{2}(\Omega)$.

The proof of this result for the case when the domain has no holes, i.e., $\Omega^{\varepsilon}=\Omega$ can be found in [26]. In the general case the proof follows analogously.

\subsection{Conforming approximation spaces via scale resolution at the boundary}

The approximation spaces $\mathcal{V}_{\varepsilon}^{N}$ defined in (47) are, when restricted to $\Omega^{\varepsilon}$, in general not $H_{\Gamma_{D}^{\varepsilon}}^{1}\left(\Omega^{\varepsilon}\right)$-conforming. We propose scale resolution at the boundary with standard FEM and use $\mathcal{V}_{\varepsilon}^{N}$ in the interior only. In Proposition 18, 
we show that such composite FE-spaces $V_{N}$ are still dense in the set of all $H_{\Gamma_{D}^{\varepsilon}}^{1}\left(\Omega^{\varepsilon}\right)$ solutions. Enforcement of the essential boundary conditions is only one reason for using scale resolution near the boundary. For problems posed on the unbounded domain the solution $u^{\varepsilon}(x)$ has a two-scale behavior and these scales are separated, but the situation is different for problems on bounded domains, since near the boundary, scale separation occurs only for special situations. For example, if the boundary is flat and aligned with the periodicity as in the case of an infinite, perforated layer, then two-scale separation occurs in the tangential direction at the boundary. In general, however, scale separation does not take place at curved or nonaligned parts of the boundary (see, e.g., [34]).

Different approaches to enforcing Dirichlet boundary conditions are possible. In the asymptotic homogenization approach $(\varepsilon \rightarrow 0)$ one constructs socalled correctors. The explicit construction of so-called "boundary correctors" (which compensate for boundary conditions violated by outer asymptotic expansions, but which are, generally, of boundary layer type) is tedious and limited to special cases, in which some geometry simplifications are assumed, as in the case when the periodic structure is aligned with the boundaries. For the general case (e.g., when the boundaries are curved and intersect the cavities of the microscopic periodic lattice) only little is known about the detailed structure of the boundary correctors (see also [34]). Such situations can be dealt with by scale resolution at the boundary. The geometry approximation comes as an additional argument for scale resolution at the boundary.

Let $\widetilde{V}_{N} \subset H^{1}\left(\Omega^{\varepsilon}\right)$ be any finite dimensional subspace (it need not satisfy the homogeneous Dirichlet boundary conditions) such that

$$
\inf _{v \in \widetilde{V}_{N}}\left\|u_{\varepsilon}-v\right\|_{H^{1}\left(\Omega_{(\varepsilon)}^{\varepsilon}\right)} \leq \delta
$$

where $\Omega_{(\varepsilon)}^{\varepsilon}:=\left\{x \in \Omega^{\varepsilon} \mid \operatorname{dist}(x, \partial \Omega)>\varepsilon\right\}$. Let $\chi \in H_{0}^{1}(\Omega) \cap W^{1, \infty}(\Omega)$ be a cut-off function such that

$$
\begin{aligned}
& \chi \equiv 1 \text { on } \Omega_{(2 \varepsilon)}:=\{x \in \Omega \mid \operatorname{dist}(x, \partial \Omega)>2 \varepsilon\}, \\
& \chi \equiv 0 \text { on } \Omega \backslash \Omega_{(\varepsilon)}=\{x \in \Omega \mid \operatorname{dist}(x, \partial \Omega) \leq \varepsilon\} .
\end{aligned}
$$

Assume further that $V_{0, \Gamma_{D}^{\varepsilon}}\left(\Omega^{\varepsilon} \backslash \Omega_{(2 \varepsilon)}\right) \subset H_{0, \Gamma_{D}^{\varepsilon}}^{1}\left(\Omega^{\varepsilon} \backslash \Omega_{(2 \varepsilon)}\right)$ is a finite dimensional subspace of $H_{0, \Gamma_{D}^{\varepsilon}}^{1}\left(\Omega^{\varepsilon} \backslash \Omega_{(2 \varepsilon)}\right):=\left\{v \in H^{1}\left(\Omega^{\varepsilon} \backslash \Omega_{(2 \varepsilon)}\right):\left.v\right|_{\Gamma_{D}^{\varepsilon}}=0\right\}$. Then we define the $H_{\Gamma_{D}^{\varepsilon}}^{1}(\Omega)$-conforming FE-space $V_{N}$ as

$$
V_{N}:=\chi \widetilde{V}_{N}+(1-\chi) V_{0, \Gamma_{D}^{\varepsilon}}\left(\Omega^{\varepsilon} \backslash \Omega_{(2 \varepsilon)}\right) .
$$

Proposition 18. Assume that the cut-off function $\chi$ introduced above satisfies:

$$
\|\chi\|_{W^{1, \infty}(\Omega)} \leq C_{\chi} \varepsilon^{-1} .
$$

Then, for each $s \in[0,1 / 2)$ there exist $C(s)=C\left(s, \Omega, C_{\chi}\right)>0$ and $C>0$ such that there holds

$$
\begin{aligned}
& \inf _{v \in V_{N}}\left\|u^{\varepsilon}-v\right\|_{H^{1}\left(\Omega^{\varepsilon}\right)} \leq C(s) \varepsilon^{-(1-s)} \delta \\
&+C \inf _{w \in V_{0, \Gamma_{D}^{\varepsilon}}\left(\Omega^{\varepsilon} \backslash \Omega_{(2 \varepsilon)}\right)}\left(\left\|\nabla\left(u^{\varepsilon}-w\right)\right\|_{L^{2}\left(\Omega^{\varepsilon} \backslash \Omega_{(2 \varepsilon)}\right)}\right. \\
&\left.+\varepsilon^{-1}\left\|\left(u^{\varepsilon}-w\right)\right\|_{L^{2}\left(\Omega^{\varepsilon} \backslash \Omega_{(2 \varepsilon)}\right)}\right) .
\end{aligned}
$$

The proof of this result for domains without holes $\left(\widehat{Q}=(-\pi, \pi)^{n}\right)$ can be found in [26], in the general case the result can be proved analogously. 
Remark 19. Let $V_{0, \Gamma_{D}^{\varepsilon}}\left(\Omega^{\varepsilon} \backslash \Omega_{(2 \varepsilon)}\right)=S_{0, \Gamma_{D}^{\varepsilon}}^{1,1}\left(\Omega^{\varepsilon} \backslash \Omega_{(2 \varepsilon)}, \mathcal{T}_{h}\right)$ be the space of piecewise linears with respect to a quasiuniform triangulation $\mathcal{T}_{h}$ of $\Omega^{\varepsilon} \backslash \Omega_{(2 \varepsilon)}$ that are zero on the boundary $\Gamma_{D}^{\varepsilon}$. Note that $\cup_{h>0} S_{0, \Gamma_{D}^{\varepsilon}}^{1,1}\left(\Omega^{\varepsilon} \backslash \Omega_{(2 \varepsilon)}, \mathcal{T}_{h}\right)$ is dense in $H_{0, \Gamma_{D}^{\varepsilon}}^{1}\left(\Omega^{\varepsilon} \backslash \Omega_{(2 \varepsilon)}\right)$ so that the infimum in the right hand side of the error estimate (50) tends to zero as $h \rightarrow 0$. If $u^{\varepsilon}$ is piecewise regular then the size of this infimum can be quantified. Assuming that $\left.u^{\varepsilon}(x)\right|_{K} \in H^{2}(K)$ for all $K \in \mathcal{T}_{h}$ we have the standard estimate [12] for the piecewise linear interpolant $u_{\text {Int }}^{\varepsilon}$ of $u^{\varepsilon}$ on $\mathcal{T}_{h}$

$$
\begin{aligned}
& h\left\|\nabla\left(u^{\varepsilon}-u_{\mathrm{Int}}^{\varepsilon}\right)\right\|_{L^{2}\left(\Omega^{\varepsilon} \backslash \Omega_{(2 \varepsilon)}\right)}+\left\|u^{\varepsilon}-u_{\mathrm{Int}}^{\varepsilon}\right\|_{L^{2}\left(\Omega^{\varepsilon} \backslash \Omega_{(2 \varepsilon)}\right)} \leq \\
& C h^{2}\left\{\sum_{K \in \mathcal{T}_{h}}\left|u^{\varepsilon}\right|_{H^{2}(K)}^{2}\right\}^{1 / 2} .
\end{aligned}
$$

For smooth coefficients $a(y), a_{0}(y)$ in (5) we have $\left\{\sum_{K \in \mathcal{T}_{h}}\left|u^{\varepsilon}\right|_{H^{2}(K)}^{2}\right\}^{1 / 2} \leq$ $C \varepsilon^{-1}$. Hence, we obtain with $w=u_{\text {Int }}^{\varepsilon}$ in (50) the following error estimate with respect to the subspace $V_{N}=\chi \widetilde{V}_{N}+(1-\chi) S_{0, \Gamma_{D}^{\varepsilon}}^{1,1}\left(\Omega^{\varepsilon} \backslash \Omega_{(2 \varepsilon)}, \mathcal{T}_{h}\right)$

$$
\inf _{v \in V_{N}}\left\|u_{\varepsilon}-v\right\|_{H^{1}\left(\Omega^{\varepsilon}\right)} \leq C_{1}(s) \varepsilon^{-(1-s)} \delta+C_{2} h / \varepsilon,
$$

with $C_{i}, i=1,2$ independent of $h$ and $\varepsilon$. Note that here $h<\varepsilon$. The space $V_{N}$ is essentially one in which scale resolution is achieved at the boundary and in which the functions from $\widetilde{V}_{N}$ are used in the interior.

\section{$5 \quad$ Numerical experiments}

We turn now to the computational investigation of the generalized FEM on bounded domains. To this end, we consider homogenization problems in one and two dimensions. The goal of the experiments is twofold. First, we would like to confirm the theoretical asymptotic convergence estimates which were presented in the previous sections, in particular the robustness of the methods with respect to $\varepsilon$. Second, we will investigate the performance of several possible choices of micro spaces $\mathcal{M}_{\varepsilon}^{\mu}$. We consider in particular the microspaces based on oversampling, on the asymptotic theory, and on Blochwave eigenfunctions.

\subsection{Microscale shape functions space $\mathcal{M}_{\varepsilon}^{\mu}(\varepsilon \widehat{Q})$ based on oversampling}

We have seen so far that collocation of the Fourier-Bochner integral kernel $\psi(x / \varepsilon, \varepsilon, t)$ at various sets of collocation points $\mathcal{N}=\left\{t_{j}\right\}_{j}$ gives dense systems of shape functions with very favorable approximation properties for elliptic homogenization problems. These shape functions are products of smooth functions in $x$ and periodic, oscillatory micro shape functions that arise as solutions of parameter dependent unit-cell problems. We present a FEM for the solution of the unit cell problem and a methodology to derive a well conditioned set of shape functions from the collocated kernels $\psi\left(x / \varepsilon, \varepsilon, t_{j}\right)$, $t_{j} \in \mathcal{N}$. This will be based on the SVD of the matrix of coefficient vectors of the Finite Element approximations to the $\phi\left(y, \varepsilon, t_{j}\right), t_{j} \in \mathcal{N}$.

To cast (27) in weak form, we define for each $\varepsilon>0$ and $t \in \mathbb{C}^{n}$ the sesquilinear form $\Phi(\varepsilon, t)[\cdot, \cdot]: H_{\mathrm{per}}^{1}(\widehat{Q}) \times H_{\mathrm{per}}^{1}(\widehat{Q}) \rightarrow \mathbb{C}$ by

$$
\begin{aligned}
& \Phi(\varepsilon, t)[\phi, v]:= \\
& \int_{\widehat{Q}} a(y) \nabla_{y}\left(\phi(y) e^{i t \cdot \varepsilon y}\right) \cdot \overline{\nabla_{y}\left(v(y) e^{i t \cdot \varepsilon y}\right)}+\varepsilon^{2} a_{0}(y) \phi(y) \overline{v(y)} d y .
\end{aligned}
$$


With this notation, the integral kernel $\phi(\cdot, \varepsilon, t) \in H_{\text {per }}^{1}(\widehat{Q})$ in the representation (24) is the weak solution of the following variational problem

$$
\begin{aligned}
& \text { Find } \phi(\cdot) \in H_{\text {per }}^{1}(\widehat{Q}) \text { such that } \\
& \Phi(\varepsilon, t)[\phi, v]=\varepsilon^{2} \int_{\widehat{Q}} \overline{v(y)} d y \quad \forall v \in H_{\mathrm{per}}^{1}(\widehat{Q}) .
\end{aligned}
$$

Let $\mathcal{N}=\left\{t_{j}: j=1, \ldots, \hat{\mu}\right\}$ be any set of collocation points in $\mathbb{C}^{n}$. Given a partition $\widehat{\mathcal{T}}$ of the unit cell $\widehat{Q}$ into intervals $K$, for an arbitrary $t_{j} \in \mathcal{N}$, compute the FE approximations

$$
\begin{aligned}
& \phi_{F E}\left(y, \varepsilon, t_{j}\right) \in S_{\text {per }}^{\hat{p}, 1}(\widehat{Q}, \widehat{\mathcal{T}}): \\
& \Phi\left(\varepsilon, t_{j}\right)\left[\phi_{F E}, v\right]=\varepsilon^{2} \int_{\widehat{Q}} \overline{v(y)} d y, \quad \forall v \in S_{\text {per }}^{\hat{p}, 1}(\widehat{Q}, \widehat{\mathcal{T}}),
\end{aligned}
$$

where $\Phi(\varepsilon, t)[\cdot, \cdot]$ is as in $(51)$ and the FE space $S_{\text {per }}^{\hat{p}, 1}(\widehat{Q}, \widehat{\mathcal{T}})$ is defined by

$$
S_{\text {per }}^{\hat{p}, 1}(\widehat{Q}, \widehat{\mathcal{T}}):=\left\{u \in H_{\mathrm{per}}^{1}(\widehat{Q}):\left.u\right|_{K} \in \mathcal{S}^{\hat{p}}(K), \forall K \in \widehat{\mathcal{T}}\right\},
$$

and $\mathcal{S}^{\hat{p}}(K)$ is a space of polynomials of degree at most $\hat{p}$ on $K$. The design of $\widehat{\mathcal{T}}, \hat{p}$ must take into account regularity of the solution. For example, if the unit cell problem has a crack, then the solution $\phi(y, \varepsilon, t) \in B_{\beta}^{2}(\hat{Q})$, the countably normed space [5], and $h p$-FEM $[37,23]$ with a geometrical mesh refinement towards the crack-tips must be employed for the solution of the unit-cell problem.

Remark 20. In the proof of the error estimate (45) the oscillating functions $\psi(x / \varepsilon, \varepsilon, t)=e^{i t \cdot x} \phi(x / \varepsilon, \varepsilon, t)$ are exact, i.e., the unit-cell solutions $\phi(y, \varepsilon, t)$ are assumed exact. The micro-scale shape functions in the definition of $\mathcal{M}_{\varepsilon}^{\mu}$ are derived from the FE approximations $\phi_{F E}(y, \varepsilon, t)$ obtained by energy projection through a FE discretization of the unit-cell problem. The stiffness matrix computations based on the generalized FE spaces $S \underline{p}\left(\Omega, \mathcal{T} ; \mathcal{M}_{\varepsilon}^{\mu}\right)$ require only moments, i.e., weighted integrals (16) of the finite element solutions $\phi_{F E}(y, \varepsilon, t)$ over the unit-cell. Careful inspection of these moments reveals that they converge quasioptimally and at the same rate as the energy norm.

Since the sesquilinear form $\Phi(\varepsilon, t)[\cdot, \cdot]$ is coercive (in the sense that a Garding inequality holds and the unit cell solution operator is injective), there exists a unique solution $\phi_{F E}\left(y, \varepsilon, t_{j}\right) \in S_{\text {per }}^{\hat{p}, 1}(\widehat{Q}, \widehat{\mathcal{T}})$ of $(53)$. In agreement with Proposition 14 , we choose $t_{j}(\mu)=j h$ where $h=1 / \sqrt{\mu}$ with $j \in \mathbb{Z}_{(\mu)}^{n}$. Notice that the values of $d$ and $\alpha$ in (38) are generally not available. Therefore, the choice of $t_{j}$ is to some extent heuristic. By Section 3.3, $\phi(y, \varepsilon, t)$ is analytic in $t$. As $\mu$ increases, the collocation points $t_{j}$ will cluster, resulting in almost linear dependence of the shape functions $\phi_{F E}\left(y, \varepsilon, t_{j}\right)$; these functions are hence not well-suited as basis for a generalized $p$-FEM. Some orthogonalization is needed to obtain a well-conditioned basis. In addition, the points $t_{j}(\mu)$ depend on $\mu$ meaning that the shape functions $\phi_{F E}\left(y, \varepsilon, t_{j}(\mu)\right)$ are not hierarchical.

We propose an oversampling, i.e., to select $\hat{\mu} \gg \mu$ sufficiently large and

$$
\mathcal{N}=\left\{t_{j}(\hat{\mu}): j=1, \ldots, \hat{\mu}\right\},
$$

and to perform an orthogonalization as follows: 
Algorithm 21. Let $\underline{N}(y)$ be a basis of $S_{\text {per }}^{\hat{p}, 1}(\widehat{Q}, \widehat{\mathcal{T}})$. Then

$$
\phi_{F E}\left(y, \varepsilon, t_{j}\right)=\Phi_{j}(\varepsilon)^{\top} \underline{N}(y), \quad j=1, \ldots, \hat{\mu} .
$$

Compute the SVD

$$
\left[\Phi_{1}(\varepsilon), \ldots, \Phi_{\hat{\mu}}(\varepsilon)\right]=\underline{U} \operatorname{diag}\left(\sigma_{1}, \ldots, \sigma_{\hat{\mu}}\right) \underline{V}^{\top}
$$

with $\sigma_{1} \geq \sigma_{2} \geq \sigma_{3} \geq \cdots \geq \sigma_{\hat{\mu}} \geq 0$ and set

$$
\mathcal{M}_{\varepsilon}^{\mu}=\operatorname{Span}\left\{\phi_{j}\left(\frac{x}{\varepsilon}, \varepsilon\right):=U_{j}^{\top} \underline{N}\left(\frac{x}{\varepsilon}\right): j=1, \ldots, \mu+1\right\},
$$

with $U_{j}$ being the $j$-th column of $\underline{U}$.

It is clear that this orthogonalization changes only the basis, but not the span of the shape functions if $\mu=\hat{\mu}$. If $\mu<\hat{\mu}$, however, the definition (55) will change the span. Nevertheless, if $\sigma_{j}<$ eps for $\mu<j \leq \hat{\mu}$ with eps of the order of machine precision, this change will be negligible. The list of significant singular values for a one dimensional model problem with $a_{0} \equiv 1, a(\cdot)$ as

\begin{tabular}{|c|c|c|}
\hline$\varepsilon=1.0 e-01$ & $\varepsilon=1.0 e-02$ & $\varepsilon=1.0 e-03$ \\
\hline$\sigma_{1}=1.017400157 e+01$ & $\sigma_{1}=8.708065522 e+00$ & $\sigma_{1}=9.651065322 e+00$ \\
\hline$\sigma_{2}=3.602041416 e+00$ & $\sigma_{2}=1.716876891 e+00$ & $\sigma_{2}=4.686737612 e-01$ \\
\hline$\sigma_{3}=3.499684680 e+00$ & $\sigma_{3}=1.517277336 e+00$ & $\sigma_{3}=3.516838658 e-02$ \\
\hline$\sigma_{4}=1.653439563 e+00$ & $\sigma_{4}=3.158726721 e-01$ & $\sigma_{4}=4.904764017 e-05$ \\
\hline$\sigma_{5}=1.118879591 e+00$ & $\sigma_{5}=1.890039200 e-02$ & $\sigma_{5}=8.322681798 e-08$ \\
\hline$\sigma_{6}=2.052151441 e-01$ & $\sigma_{6}=2.906576569 e-05$ & $\sigma_{6}=1.003629032 e-11$ \\
\hline$\sigma_{7}=4.486387147 e-04$ & $\sigma_{7}=2.709646458 e-09$ & $\sigma_{7}=7.670974077 e-15$ \\
\hline$\sigma_{8}=7.805541440 e-07$ & $\sigma_{8}=2.782052676 e-13$ & $\sigma_{8}=7.669801103 e-18$ \\
\hline$\sigma_{9}=2.429807212 e-11$ & $\sigma_{9}=1.612941949 e-17$ & $\sigma_{9}=5.661242396 e-18$ \\
\hline$\varepsilon=1.0 e-04$ & $\varepsilon=1.0 e-05$ & $\varepsilon=1.0 e-06$ \\
\hline$\sigma_{1}=9.651024285 e+00$ & $\sigma_{1}=9.650620918 e+00$ & $\sigma_{1}=9.856848581 e+00$ \\
\hline$\sigma_{2}=4.641386272 e-02$ & $\sigma_{2}=4.640937839 e-03$ & $\sigma_{2}=4.641062782 e-04$ \\
\hline$\sigma_{3}=3.481845540 e-04$ & $\sigma_{3}=3.481393137 e-06$ & $\sigma_{3}=3.533916622 e-08$ \\
\hline$\sigma_{4}=4.898472869 e-08$ & $\sigma_{4}=4.898386017 e-11$ & $\sigma_{4}=4.907798256 e-14$ \\
\hline$\sigma_{5}=8.316984046 e-12$ & $\sigma_{5}=4.464187517 e-14$ & $\sigma_{5}=2.457293272 e-14$ \\
\hline
\end{tabular}
in (56) and different $\varepsilon$ scales is presented in Table 1. The largest singular values appear to be proportional to some powers of $\varepsilon$. Span $\left\{\psi\left(\cdot / \varepsilon, \varepsilon, t_{j}\right)\right\}$ is

Table 1. The largest singular values $\sigma_{j}$ of the coefficient matrix with respect to the shape functions $\left\{\phi_{F E}\left(\cdot, \varepsilon, t_{j}\right), j=0,1, \ldots, \hat{\mu}\right\}_{t_{j}=j / \sqrt{\hat{\mu}}}, \hat{\mu}=64$

practically independent on the choice of the collocation points. Therefore the precise choice of $t_{j}$ will not matter much, as long as with increasing $\mu$ they cover the interval $[-\sqrt{\mu}, \sqrt{\mu}]^{n}$ and are spaced as $1 / \sqrt{\mu}$ by Proposition 14 . Figure 2 presents the shape functions $\left\{\phi_{j}(y, \varepsilon)\right\}_{j=1}^{\mu}$ obtained with Algorithm 21 for the one-dimensional problem with $\widehat{Q}=(-\pi, \pi), a_{0} \equiv 1, a(\cdot)$ as in (56), $\varepsilon=0.001$ and, based on Proposition 14, the set of collocation points $\mathcal{N}=\left\{t_{j}(\hat{\mu})=j / \sqrt{\hat{\mu}}: j=0, \ldots, \hat{\mu}, \hat{\mu}=64\right\}$. In this case the number of $j$ such that the corresponding singular values $\sigma_{j}>$ eps $=10^{-10}$ is $\mu=5$. Hence the orthogonalization has also reduced the number of micro shape functions substantially. We clearly see the substantial reduction of degrees of freedom due to the almost linear dependence of the $\phi_{F E}\left(y, \varepsilon, t_{j}\right)$ and the low 
regularity of these shape functions at the jumps of $a(\cdot)$ at $y= \pm \pi / 2$. Note also that, unlike the kernels $\phi\left(y, \varepsilon, t_{j}\right), \phi_{j}(y, \varepsilon)$ are piecewise polynomials.

$$
a(y)=\left\{\begin{array}{cl}
10 & \text { if }|y| \leq \frac{\pi}{2}, \\
1 & \text { else }
\end{array}\right.
$$

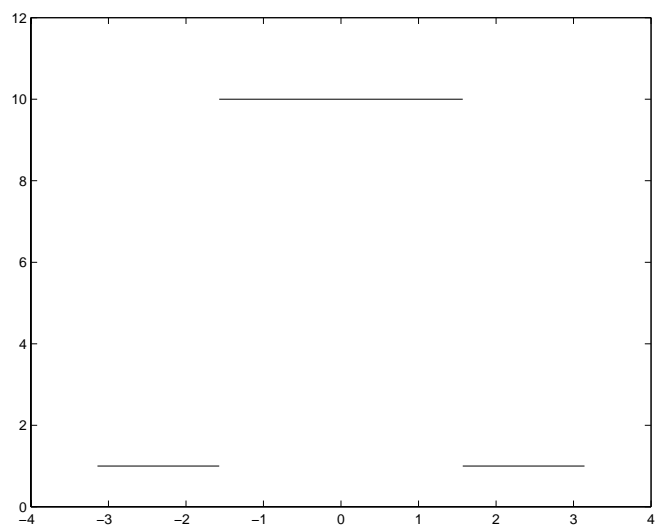

Fig. 1. The coefficient $a(\cdot)$.

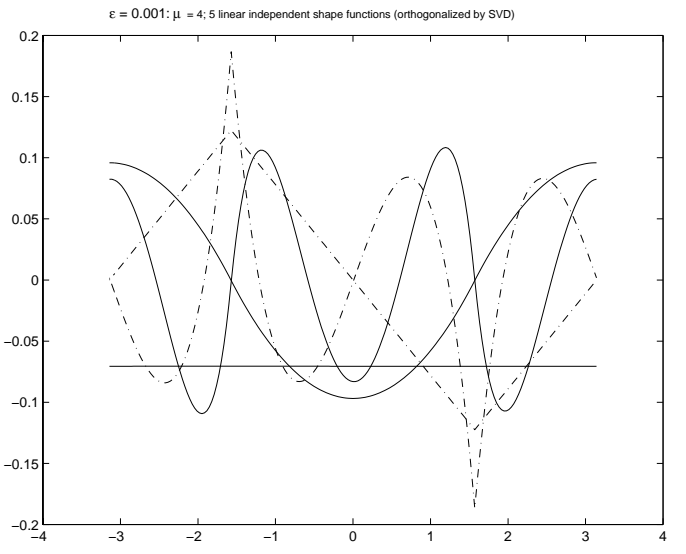

Fig. 2. $\phi_{j}(\cdot, \varepsilon), j=1, \ldots, \mu$

Remark 22. In numerical experiments we found that Algorithm 21 is very robust with respect to the choice of collocation points. After the SVD the first shape functions associated with the largest singular values are practically independent of the number and the choice of $t_{j}$. The shape functions $\phi_{j}(\cdot, \varepsilon)$ resulting from Algorithm 21 are therefore, at least numerically, hierarchical, and enable hierarchic modeling of problems with microstructure.

\section{2 gFEM based on oversampling in the one-dimensional case}

We consider the following model problem in $\Omega:=(-1,1)$ : let $f(\cdot) \in L^{2}(\Omega)$ and $\varepsilon>0$ fixed. Denote by $u^{\varepsilon}(\cdot) \in H_{0}^{1}(\Omega)$ the weak solution of the following 
boundary value problem

$$
\begin{aligned}
-\frac{d}{d x}\left(a\left(\frac{x}{\varepsilon}\right) \frac{d u^{\varepsilon}}{d x}(x)\right)+a_{0}\left(\frac{x}{\varepsilon}\right) u^{\varepsilon}(x) & =f(x) \text { in } \Omega, \\
u^{\varepsilon}( \pm 1) & =0,
\end{aligned}
$$

with

$$
a(y)=\left\{\begin{array}{cl}
10 & \text { if }|y| \leq \frac{\pi}{2} \\
1 & \text { else },
\end{array}\right.
$$

and $a_{0}(y) \equiv 1$. We implemented the generalized $p$-FEM described in the previous section for (57) with $a(\cdot)$ as in (56) and absolute terms $a_{0} \equiv 1$, $a_{0} \equiv 0$, respectively. The exact solution $u^{\varepsilon}(x)$ is in general piecewise analytic but non-polynomial on the microscale. We used in all our computations the orthonormalized micro shape functions $\phi_{j}(y, \varepsilon)$ in $(55)$ from the unit cell problem with absolute term $a_{0} \equiv 1$.

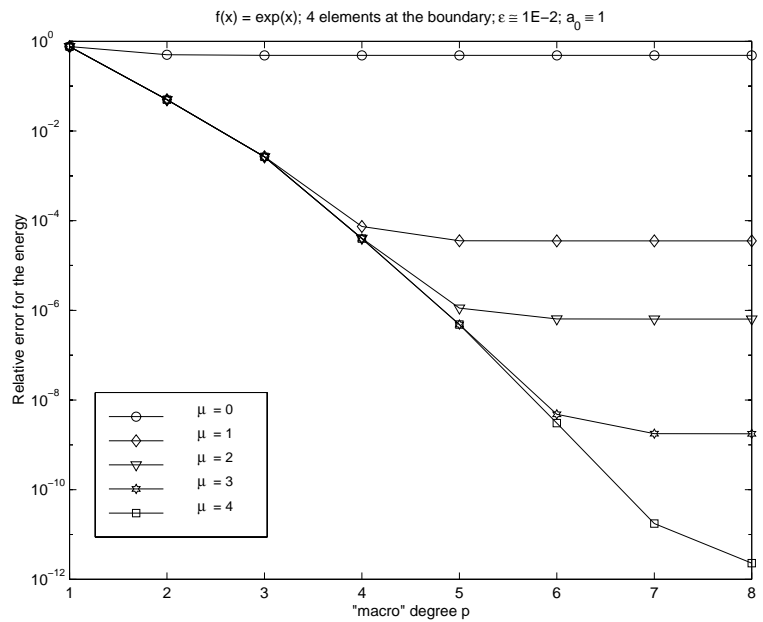

Fig. 3. Exponential rate of convergence for the FE energy with $\mathcal{M}_{\varepsilon}^{\mu}$ based on oversampling. $f(x)=\exp (x)$.

Figure 3 shows the convergence of the generalized $p$-FEM for $a_{0} \equiv 1, f(x)=$ $\exp (x)$ and $\varepsilon \cong 10^{-2}$ when $p$ is increased on a fixed mesh $\mathcal{T}=\mathcal{T}_{0} \cup \mathcal{T}_{b}$ with $\mathcal{T}_{b}$ covering 4 periods of length $2 \pi \varepsilon$ at each boundary point for various values of $\mu$. The curves corresponding to $\mu=0$ show the error when only macroscopic shape functions, i.e. global polynomials, are used.

We see that for fixed $\mu>0$ and increasing $p$, first exponential convergence is apparent, however a saturation occurs at a $p$-level which depends on the micro degree $\mu$. Exponential convergence requires therefore the joint increase of the micro degree $\mu$ with the macro degree $p$.

In Figure 4 we show analogous results for $a_{0} \equiv 0, f(x)=\exp (x)$ (with respect to the same mesh) and different microscales $\varepsilon$, varying from $\cong 10^{-1}$ down to $\cong 10^{-6}$. We note that for $\mu=1$ and for $\varepsilon \cong 10^{-1}$ a very slow convergence is apparent - here the scales are resolved, but the low solution regularity stalls the spectral convergence. As before, one can see from the results in Figure 5 that keeping $p$ fixed and increasing $\mu$ does not lead to exponential 

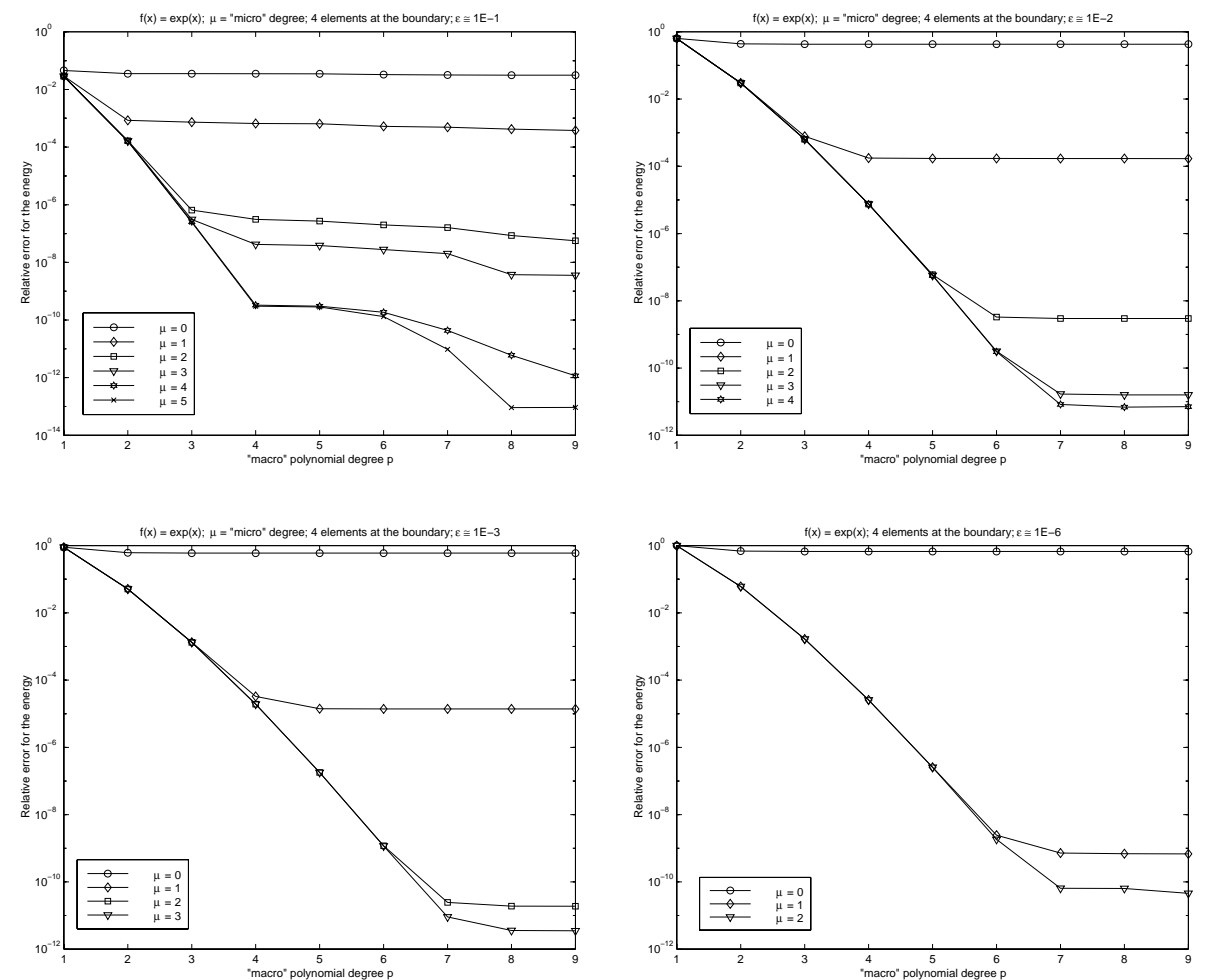

Fig. 4. Convergence rate for fixed micro degree $\mu$ and increasing macro polynomial degree $p$. The system of micro shape functions $\mathcal{M}_{\varepsilon}^{\mu}$ is based on the oversampling procedure in Algorithm 21 and $f(x)=\exp (x)$.

convergence. Rather, Figures 3, 4, 5 show again that $\mu$ must be increased together with $p$.

\subsection{Quasi 1-D Case}

The generalized FEM with micro scale shape functions $\mathcal{M}_{\varepsilon}^{\mu}$ derived by oversampling the Fourier-Bochner integral kernel has been implemented for two dimensional problems. Our gFEM implementation is based on Concepts-1.4, an object oriented programming framework for general elliptic problems in $\mathrm{C}++[24]$. We illustrate here the performance of the gFEM considering first the (quasi 1-d) model problem

$$
\begin{aligned}
-\nabla \cdot\left(a\left(\frac{x}{\varepsilon}\right) \nabla u^{\varepsilon}(x)\right) & =f(x) \quad \text { in } \Omega:=(0,1)^{2} \\
\left.u^{\varepsilon}\right|_{\Gamma_{D}} & =0 \quad \text { on } \Gamma_{D}:=\left\{x_{1}=0\right\} \cup\left\{x_{1}=1\right\} \\
\left.n \cdot a\left(\frac{x}{\varepsilon}\right) u^{\varepsilon}(x)\right|_{\Gamma_{N}} & =0 \quad \text { on } \Gamma_{N}:=\partial \Omega \backslash \Gamma_{D},
\end{aligned}
$$

where $a(y)=\cos \left(y_{1}\right)+2, y=\left(y_{1}, y_{2}\right) \in \widehat{Q}:=(0,2 \pi)^{2}$ and $f(x) \equiv 1$. The implementation is completely general, but since we know for this problem 


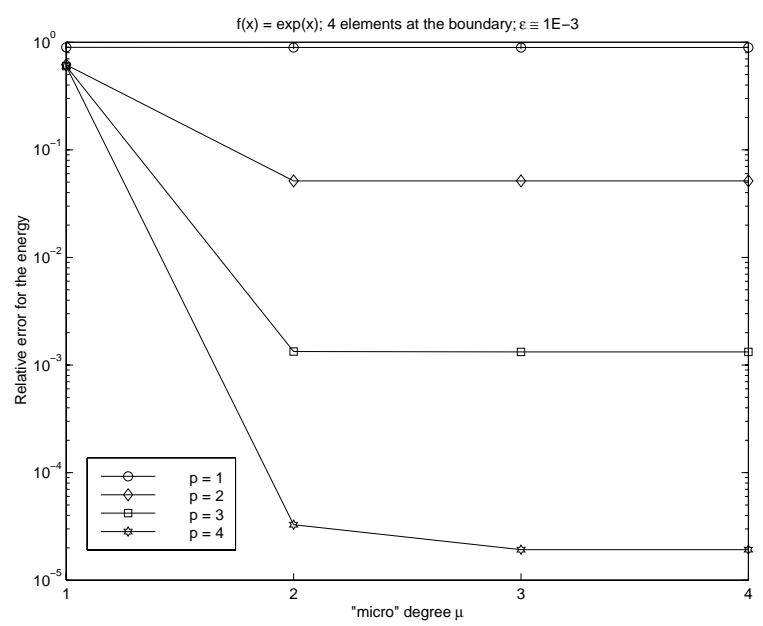

Fig. 5. Convergence rate for fixed macro polynomial degree $p$ and increasing micro degree $\mu\left(\mathcal{M}_{\varepsilon}^{\mu}\right.$ based on oversampling). $f(x)=\exp (x)$.

the exact solution

$$
u^{\varepsilon}(x)=-\int_{0}^{x_{1}} \frac{t}{a(t / \varepsilon)} d t+\frac{\int_{0}^{1} \tau / a(\tau / \varepsilon) d \tau}{\int_{0}^{1} 1 / a(\tau / \varepsilon) d \tau} \int_{0}^{x_{1}} \frac{1}{a(t / \varepsilon)} d t
$$

it is reasonable to investigate the numerical performance for such solutions. The macro mesh $\mathcal{T}$ used in all our experiments is shown in Figure 6. It consists of four interior elements of size $(1-4 \pi \varepsilon) / \varepsilon$ and two layers of elements in a tubular neighborhood of $\partial \Omega$ covering two periods in the normal direction to $\partial \Omega$. The boundary elements are either elements near corners of the size of one micro cell or elongated elements along edges that are of size $O(\varepsilon)$ in the normal direction to the boundary. We recall that the macro polynomial degree has to be increased together with the number of micro shape functions in order to achieve convergence, thus we have successively and simultaneously increased $p$ and the micro degree $\mu$ in all elements $K \in \mathcal{T}$ such that $p=\mu+1$. The relative error in the energy for increasing macro polynomial degree $p$ and micro degree $\mu$ such that $p=\mu+1, \mu=0,1,2,3$, and for different $\varepsilon$ scales is presented in Table 2.

\begin{tabular}{|r|rrrrr|}
\hline$p$ & $\varepsilon \cong 10^{-2}$ & $\varepsilon \cong 10^{-3}$ & $\varepsilon \cong 10^{-4}$ & $\varepsilon \cong 10^{-5}$ & $\varepsilon \cong 10^{-6}$ \\
\hline 1 & 0.150836 & 0.309959 & 0.346179 & 0.350048 & 0.350437 \\
\hline 2 & 0.079917 & 0.133956 & 0.133974 & $1.714673 \mathrm{e}-05$ & $2.229294 \mathrm{e}-06$ \\
\hline 3 & $1.202447 \mathrm{e}-05$ & $2.320999 \mathrm{e}-06$ & $7.504589 \mathrm{e}-07$ & $5.895692 \mathrm{e}-07$ & $5.734377 \mathrm{e}-07$ \\
\hline 4 & $1.874951 \mathrm{e}-07$ & $4.898619 \mathrm{e}-07$ & $5.629935 \mathrm{e}-07$ & $5.710097 \mathrm{e}-07$ & $5.715827 \mathrm{e}-07$ \\
\hline
\end{tabular}

Table 2. Relative error in the energy for gFEM with $p=\mu+1$. 


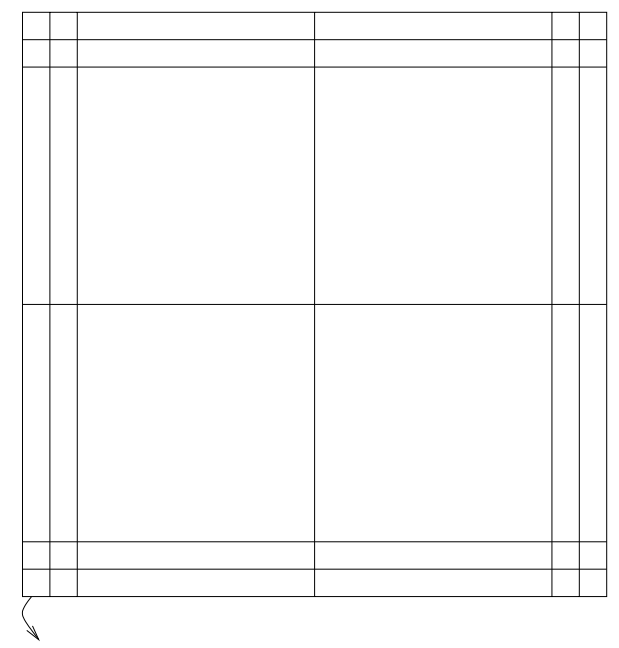

Fig. 6. The macro mesh $\mathcal{T}$ (not at scale) for $2-\mathrm{D}$ calculations.

\subsection{2-D Problem}

In this section we analyse the performance of the gFEM for a genuinely twodimensional model problem

$$
\begin{aligned}
-\nabla \cdot\left(a\left(\frac{x}{\varepsilon}\right) \nabla u^{\varepsilon}(x)\right)+a_{0}\left(\frac{x}{\varepsilon}\right) u^{\varepsilon}(x) & =f(x) \quad \text { in } \Omega:=(0,1)^{2} \\
u^{\varepsilon} \mid \partial \Omega & =0 \quad \text { on } \partial \Omega,
\end{aligned}
$$

with

$$
f(x)=10 x_{1}+10 x_{2}, \quad a(y)=6+\cos \left(y_{1}\right)+\cos \left(y_{2}\right), \quad \text { and } a_{0}(y) \equiv 1 .
$$

Since the exact solution is not available, we compare our numerical results with the results obtained by doing scale resolution via standard FE discretizations. The unit-cell problem has been solved numerically by standard FEM. The unit-cell triangulation $\widehat{\mathcal{T}}$ was obtained by a three level uniform refinement of the unit-cell domain and the polynomial degree in each element was $\hat{p}=2$. The collocation set in our computations here was $\mathcal{N}=\left\{\left(10^{i}, 10^{j}\right)\right.$ : $-4 \leq i, j \leq 2\}$. After the SVD of the matrix of coefficient vectors of the discrete unit-cell solutions we select $\mathcal{M}_{\varepsilon}^{\mu}:=\operatorname{Span}\left\{\phi_{i}(x / \varepsilon ; \varepsilon): i=0,1, \ldots, \mu\right\}$ as the space of resulting shape functions corresponding to the first $\mu$ largest singular values. The unit-cell computations represent an independent subroutine of our gFEM implementation. For the setting of the elemental stiffness matrices only the monomial weighted integrals of the form

$$
\int_{\widehat{Q}} a(y) y_{1}^{r} y_{2}^{s} D_{y}^{\alpha} \phi_{m}(y ; \varepsilon) D_{y}^{\beta} \phi_{n}(y ; \varepsilon) d y, \quad|\alpha|,|\beta| \leq 1
$$

are needed, thus the gFEM can handle other choices of micro scale spaces $\mathcal{M}_{\varepsilon}^{\mu}$ as well. The micro length scale $\varepsilon$ in our computations here is $\varepsilon=1 /(40 \pi)$, that means that there are 20 periods of length $2 \pi \varepsilon$ along one side of $\partial \Omega$. The relatively coarse macro mesh $\mathcal{T}$ for the gFEM is as illustrated in Figure 6. We used a constant macro polynomial degree $p_{K}=4$ for all $K \in \mathcal{T}$ and the micro degree was also constant $\mu_{K}=4$ in all elements. The number of degrees of freedom for the gFEM computations was $\# D O F \cong 1000$, whereas for the standard FEM the number of degrees of freedom was \#DOF $\cong 40000$. 
We emphasize that the number of degrees of freedom and the CPU time by the gFEM is independent of the micro length scale $\varepsilon$. Computation for $\varepsilon \cong 10^{-6}$, for example, costs the same work as $\varepsilon=10^{-2}$.

We present at the top of Figure 7 the first component of the energy flux $a(x / \varepsilon) \nabla u^{\varepsilon}$ obtained by the generalized FEM based on oversampling and compare it with the results obtained by the standard FEM (bottom). Figure 8 displays the second component of $a(x / \varepsilon) \nabla u^{\varepsilon}$ obtained with gFEM and the standard FEM and finally, in Figure 9 we compare the solution plots. We see that the gFEM is able to accurately capture all the solution characteristics with significantly less degrees of freedom than the standard FEM.
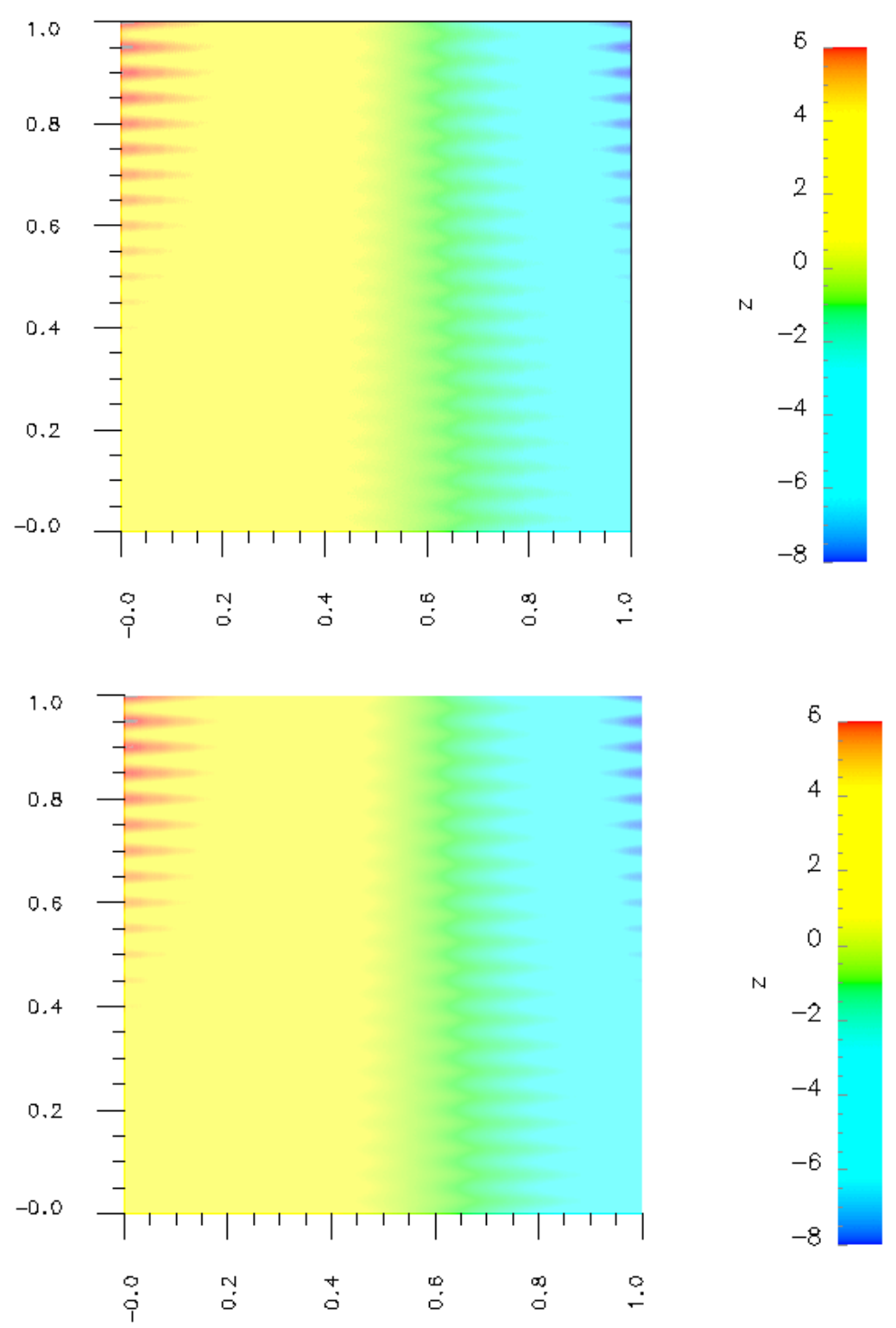

Fig. 7. The first component of the flux $a(x / \varepsilon) \nabla u^{\varepsilon}$ obtained by the generalized FEM based on oversampling (top) and by scale resolution (bottom); $\widehat{Q}=(0,2 \pi)^{2}$, $\varepsilon=1 /(40 \pi)$. 


\subsection{Microscale space based on Bloch Wave eigenmodes}

\section{Bloch waves}

One possible way to generalize the ideas of Fourier analysis to the study of non-homogeneous periodic media is the Bloch transform. The generalized eigenfunctions are known as classical Bloch waves and were introduced in solid state physics in the context of propagation of electrons in a crystal, [9]. The starting point is the spectral analysis of the operator

$$
L\left(\partial_{y}\right):=-\nabla_{y} \cdot\left(a(y) \nabla_{y}\right) .
$$

We define the shifted operator $L\left(\partial_{y} ; \eta\right)$ associated with $L\left(\partial_{y}\right)$ by

$$
L\left(\partial_{y} ; \eta\right):=-\left(\nabla_{y}+i \eta\right) \cdot\left[a(y)\left(\nabla_{y}+i \eta\right)\right] .
$$

Note that $L\left(\partial_{y} ; \eta\right)$ represents the principal part of the unit cell operator $(27)$. We attach to the shifted operator a family of spectral problems parametrized by $\eta \in \mathbb{R}^{n}:$ Find $\lambda(\eta) \in \mathbb{R}$ and $\phi(\cdot ; \eta) \in H_{\text {per }}^{1}(\widehat{Q})$ not identically zero such that

$$
L\left(\partial_{y} ; \eta\right) \phi(\cdot ; \eta)=\lambda(\eta) \phi(\cdot ; \eta) .
$$

With these notations, the unit-cell operator in $(27)$ can be written as $L\left(\partial_{y} ; \varepsilon t\right)+$ $\varepsilon^{2} a_{0}(\cdot)$. Then $\psi(\cdot ; \eta):=e^{i \eta \cdot y} \phi(\cdot ; \eta)$ solve the parameter dependent spectral problems

$$
\begin{aligned}
L\left(\partial_{y}\right) \psi(y ; \eta) & =\lambda(\eta) \psi(y ; \eta) \quad \text { in } \mathbb{R}^{n} \\
\psi(y+2 \pi k ; \eta) & =e^{2 \pi i k \cdot \eta} \psi(y), \quad \forall k \in \mathbb{Z}^{n}, y \in \mathbb{R}^{n} .
\end{aligned}
$$

The eigenfunctions $\psi(y ; \eta)$ are the so called Bloch waves and represent the natural generalization of the Fourier waves, the eigenfunctions in the case of constant coefficient, to the case of periodic media. It is also easy to see that the Bloch eigenvalues in (60) are $\mathbb{Z}^{n}$-periodic and the eigenmodes are shifted-periodic in the second variable in the sense that for all $k \in \mathbb{Z}^{n}$ there holds holds $\phi(\cdot ; \eta+k)=e^{-i k \cdot y} \phi(\cdot ; \eta)$. It is therefore enough to study them for $\eta \in[0,1)^{n}$, the first Brillouin zone.

The property of the shifted operator to depend polynomially on $\eta$ does not imply regularity of the Bloch eigenvalues and eigenvectors with respect to this parameter. In fact, it can be shown [15] that the eigenvalues are Lipschitz functions of $\eta$, and in general exhibit singularities. The first eigenvalue $\lambda_{1}(\cdot)$ is analytic and geometrically simple near $\eta=0$. In addition, the first eigenfunction can be chosen so that it depends analytically on $\eta$ in a small neighborhood of the origin. More detailed regularity properties of the first Bloch eigenvalue and eigenmode are listed below:

Proposition 23. The first Bloch eigenvalue is stationary at $\eta=0$

$$
\nabla_{\eta} \lambda_{1}(0)=0 .
$$

Further, the second derivative (or in higher dimensions the Hessian matrix) of $\lambda_{1}(\cdot)$ at $\eta=0$ is the homogenized coefficient (matrix) [13]

$$
\frac{1}{2} \frac{\partial^{2} \lambda_{1}}{\partial \eta_{i} \partial \eta_{j}}(0)=\frac{1}{(2 \pi)^{n}} \Phi_{0}\left(\chi_{1 ; i}+y_{i}, \chi_{1 ; j}+y_{j}\right)
$$

whereas the first order derivatives of the first Bloch mode at $\eta=0$ give the correction terms $\chi_{1 ; j}(\cdot)$

$$
\frac{\partial \phi_{1}}{\partial y_{j}}(\cdot ; 0)=i \frac{1}{(2 \pi)^{n / 2}} \chi_{1 ; j}(\cdot),
$$


where $\chi_{1 ; j}(\cdot) \in H_{\mathrm{per}}^{1}(\widehat{Q})$ are solutions to

$$
\chi_{1 ; j}(\cdot) \in H_{\mathrm{per}}^{1}(\widehat{Q}), \int_{\widehat{Q}} \chi_{1 ; j}(y) d y=0: \quad L\left(\partial_{y}\right)\left(\chi_{1 ; j}+y_{j}\right)=0 \quad \text { in } \widehat{Q} .
$$

A proof of this proposition can be found in [15].

The next step is to introduce the Bloch eigenvalues $\left\{\lambda_{m}^{\varepsilon}(t)\right\}_{m=1}^{\infty}$ and the eigenvectors $\left\{\phi_{m}^{\varepsilon}(x ; t)\right\}_{m=1}^{\infty}$ at $\varepsilon$ scale. By diagonalizing the principal part of the second order elliptic operator (18)

$$
-\nabla_{x} \cdot\left(a\left(\frac{x}{\varepsilon}\right) \nabla_{x}\left(e^{i t \cdot x} \phi_{m}^{\varepsilon}(x ; t)\right)\right)=\lambda_{m}^{\varepsilon}(t) \phi_{m}^{\varepsilon}(x ; t)
$$

the differential equation $L\left(\varepsilon^{-1} \partial_{x}\right) u^{\varepsilon}=f$ can be transformed into a set of algebraic equations for the Bloch transforms. The energy of $u^{\varepsilon}$ contained in all Bloch modes except the first one goes to zero when $\varepsilon \rightarrow 0$. Therefore, the first Bloch mode is essential for the asymptotic limit. It turns out [1] that the first Bloch mode transform tends to the usual Fourier transform in the $L^{2}\left(\mathbb{R}^{n}\right)$ topology as $\varepsilon \rightarrow 0$. Thus, the Bloch waves representing periodic medium tend to Fourier waves representing the homogenized medium. By a scaling argument, for $t \in \varepsilon^{-1}(-1 / 2,1 / 2)^{n}$, the Bloch eigenvalues and eigenfunctions at $\varepsilon$ scale are found to be related to $\lambda_{m}(\eta), \phi_{m}(\cdot ; \eta)$ in $(60)$ as follows:

$$
\lambda_{m}^{\varepsilon}(t)=\varepsilon^{-2} \lambda_{m}(\varepsilon t), \quad \phi_{m}^{\varepsilon}(x ; t)=\phi_{m}\left(\frac{x}{\varepsilon} ; \varepsilon t\right) .
$$

The Bloch waves $e^{i t \cdot x} \phi_{m}^{\varepsilon}(x ; t)$ at $\varepsilon$ scale provide now the spectral resolution of $L\left(\varepsilon^{-1} \partial_{x}\right)$. For $g \in L^{2}\left(\mathbb{R}^{n}\right)$ arbitrary, the $m$-th Bloch coefficient of $g$ is defined as

$$
\hat{g}_{m}^{\varepsilon}(t):=\varepsilon^{-n / 2} \int_{\mathbb{R}^{n}} g(x) e^{-i t \cdot x} \overline{\phi_{m}^{\varepsilon}(x ; t)} d x .
$$

Analogously to Fourier theory, inversion formula and a generalized Plancherel identity hold:

$$
\begin{aligned}
g(x) & =\varepsilon^{n / 2} \int_{\varepsilon^{-1}\left(-\frac{1}{2}, \frac{1}{2}\right)^{n}} \sum_{m=1}^{\infty} \hat{g}_{m}^{\varepsilon}(t) e^{i t \cdot x} \phi_{m}^{\varepsilon}(x ; t) d t \\
\varepsilon^{-n} \int_{\mathbb{R}^{n}} g(x) \overline{h(x)} d x & =\int_{\varepsilon^{-1}\left(-\frac{1}{2}, \frac{1}{2}\right)^{n}} \sum_{m=1}^{\infty} \hat{g}_{m}^{\varepsilon}(t) \overline{\hat{h}_{m}^{\varepsilon}(t)} d t, \quad \forall g, h \in L^{2}\left(\mathbb{R}^{n}\right) .
\end{aligned}
$$

Due to these results the differential equation $L\left(\varepsilon^{-1} \partial_{x}\right) u^{\varepsilon}(x)=f(x)$ in $\mathbb{R}^{n}$ transforms into an infinite algebraic system for the Bloch coefficients of $u^{\varepsilon}$

$$
\hat{f}_{m}^{\varepsilon}(\xi)=\lambda_{m}^{\varepsilon}(\xi) \hat{u}_{m}^{\varepsilon}(\xi), \quad \forall m \geq 1, \forall \xi \in \varepsilon^{-1}(-1 / 2,1 / 2)^{n} .
$$

Furthermore, when $\varepsilon \rightarrow 0$, all Bloch transforms except the first one converge to $0[15]$.

Proposition 24. Let

$$
w^{\varepsilon}(x)=\varepsilon^{n / 2} \int_{\varepsilon^{-1}(-1 / 2,1 / 2)^{n}} \sum_{m \geq 2} \hat{u}_{m}^{\varepsilon}(\xi) e^{i \xi \cdot x} \phi_{m}^{\varepsilon}(x ; \xi) d \xi .
$$

Then, there exists a constant $C>0$ such that $\left\|w^{\varepsilon}\right\|_{L^{2}\left(\mathbb{R}^{n}\right)} \leq C \varepsilon$. 


\section{Generalized FEM based on Bloch waves}

From the classical theory on homogenization $[7,8,35,13]$ based on asymptotic analysis as $\varepsilon \rightarrow 0$ it is known that the solutions $u^{\varepsilon} \in H_{0}^{1}(\Omega)$ to

$$
\begin{aligned}
L\left(\varepsilon^{-1} \partial_{x}\right) u^{\varepsilon}=f & \text { in } \Omega \\
\left.u^{\varepsilon}\right|_{\partial \Omega}=0 & \text { on } \partial \Omega
\end{aligned}
$$

converge, as $\varepsilon \rightarrow 0$, to a limit $u^{0}$ that solves the so-called homogenized problem

$$
\begin{aligned}
-\nabla \cdot\left(A \nabla u^{0}\right) & =f \quad \text { in } \Omega \\
\left.u^{0}\right|_{\partial \Omega}=0 & \text { on } \partial \Omega,
\end{aligned}
$$

where the homogenized coefficients $A=\left\{A_{i j}\right\}_{i, j}$ are given by $A_{i j}=\Phi_{0}\left(\chi_{1 ; i}+\right.$ $\left.y_{i}, \chi_{1 ; j}+y_{j}\right)$, see also (62). We see that the homogenized coefficients are not only means over the initial ones, but contain an additional term coming from the microstructure. This term is computed using the solutions $\chi_{1 ; i}$ in (64) on the unit cell.

More precisely, $\left\|u^{\varepsilon}-u^{0}\right\|_{L^{2}(\Omega)} \rightarrow 0$ as $\varepsilon \rightarrow 0$, however $\left\|u^{\varepsilon}-u^{0}\right\|_{H^{1}(\Omega)} \not \rightarrow 0$, as $\varepsilon \rightarrow 0$, since the small scale features of the solutions are lost by this averaging process. To improve the convergence, one needs the correctors

$$
\left\|u^{\varepsilon}(x)-u^{0}(x)-\varepsilon \sum_{i} \frac{\partial u^{0}}{\partial x_{i}}(x) \chi_{1 ; i}\left(\frac{x}{\varepsilon}\right)\right\|_{H^{1}(\Omega)} \rightarrow 0 \quad \text { as } \varepsilon \rightarrow 0 .
$$

The homogenized limit $u^{0}$ is independent of $\varepsilon$ and can be well approximated by standard polynomial FE spaces. Due to (66) it would be of interest to consider for the choice of the micro-scale space $\mathcal{M}^{\mu}$ the system of correctors $\left\{\chi_{1 ; i}(y)\right\}_{i}$ in (64) or, due to the fact that the first Bloch eigenmode $\phi_{1}(\cdot ; \eta)$ is analytic at 0 and its first order derivatives with respect to $\eta$ at $\eta=0$ are precisely the correctors $\chi_{1 ; i}(\cdot)$, the system of micro-scale shape functions derived by sampling the first Bloch eigenmode in a collocation set refined geometrically at $\eta=0$.

We studied the performance of the generalized FEM when the system of microscale shape functions was derived by sampling the first Bloch eigenmode in a collocation set refined geometrically at $\eta=0$. We discretize the unitcell by the FE space $S_{\text {per }}^{\hat{p}, 1}(\widehat{Q}, \widehat{\mathcal{T}})$ and then solve a parameter dependent class of generalized eigenvalue problems parametrized with respect to $\eta$ in the collocation set $\mathcal{N}_{B}=\left\{\eta_{j}=10^{-j}, j=0, \ldots, \hat{\mu}\right\}$.

Let us denote by a superscript $B$ (in order to distinguish them from the generalized shape functions $\phi_{j}(\cdot, \varepsilon)$ introduced in (55)) the Bloch shape functions $\phi_{i}^{B}(y), i=0,1, \ldots$, which are obtained by orthogonalizing the coefficient matrix of the first Bloch eigenfunctions with respect to $\eta$ in a collocation set $\mathcal{N}_{B}$. We define the generalized FE space

$$
S_{0}^{\underline{p}}\left(\Omega, \mathcal{T} ; \mathcal{M}_{\varepsilon, B}^{\underline{\mu}}\right)=\left(S \underline{p}(\Omega, \mathcal{T}) \otimes_{\mathcal{T}} \mathcal{M}_{\varepsilon, B}^{\underline{\mu}}(\Omega, \mathcal{T})\right) \cap H_{0}^{1}(\Omega)
$$

where

$$
\mathcal{M}_{\varepsilon, B}^{\mu}=\operatorname{Span}\left\{\phi_{i}^{B}\left(\frac{x}{\varepsilon}\right): i=0, \ldots, \mu \ll \hat{\mu}\right\} .
$$

The system of Bloch shape functions obtained from the orthogonalization procedure by refining the collocation set geometrically towards $\eta=0$ and selecting the first Bloch eigenfunction for each sampling point is presented in Figure 10. The collocation set is $\mathcal{N}_{B}=\left\{\eta_{j}=10^{-j}, j=0, \ldots, \hat{\mu}\right\}$, with 
$\hat{\mu}=10$, and the unit-cell problems discretization is based on the FE space $S_{\text {per }}^{\hat{p}, 1}(\widehat{Q}, \widehat{\mathcal{T}})$ of piecewise polynomials of degree $\hat{p}=8$.

The performance of the generalized two-scale space $S_{0}^{\underline{p}}\left(\Omega, \mathcal{T} ; \mathcal{M}_{\varepsilon, B}\right)$ for the model problem (57) with right hand side $f(x)=\exp (x)$ has been investigated numerically. The macro mesh $\mathcal{T}=\mathcal{T}_{b} \cup \mathcal{T}_{0}$ consists of boundary elements $K \in \mathcal{T}_{b}$ of size $O(\varepsilon)$ and one interior element $K \in \mathcal{T}_{0}$ of size $O(1)$. The boundary mesh $\mathcal{T}_{b}$ covers 4 periods of length $2 \pi \varepsilon$ at each boundary point. The micro degree $\mu_{K}=\mu$ is successively increased in the interior element $K \in \mathcal{T}_{0}$ whereas $\mu_{k}=0$ in all boundary elements $K \in \mathcal{T}_{b}$. We choose a constant macro degree $p_{K}=p$ in all elements of $\mathcal{T}$ and we vary $p$ from $p=1$ up to $p=9$.

The relative error in the energy versus the macro degree $p$ is shown in Figure 11 for different micro degrees $\mu$ and for $\varepsilon$ scales varying from $10^{-2}$ down to $10^{-5}$. We observe that when increasing $\mu$ from $\mu=0$ to $\mu=3$ only a very slow convergence occurs (for $\varepsilon \cong 10^{-4}$ and $\varepsilon \cong 10^{-5}$, the error curves corresponding to $\mu=0,1$ and 2 are practically on top of each other). Compared to the performance of the gFEM with $\mathcal{M}_{\varepsilon}^{\mu}$ based on oversampling in Figure 4 , the micro scale shape functions derived from the first collocated Bloch eigenmode that correspond to the largest singular values are not able to represent correctly the micro structure of the solution. Exponential convergence is observed first when the micro degree $\mu \geq 3$ and for each $\mu$ a saturation occurs at $p$ values depending on $\mu$ and $\varepsilon$. To achieve exponential convergence the macro polynomial degree has to be increased together with $\mu$. Comparing the error plots for the different $\varepsilon$ scales at fixed $\mu=5$ we observe that the saturation occurs earlier for smaller $\varepsilon$. The convergence rate is therefore not robust.

The two-scale gFEM based on $\mathcal{M}_{\varepsilon, B}^{\mu}$ is quite sensitive with respect to the choice of the collocation set and selection of the eigenmodes. Our next experiment suggests that if microspaces based on Bloch waves are used in the gFEM, it is sufficient to use the first Bloch eigenmode only. In Figure 12 we plot the relative error in the energy versus $p$ for different $\mu$ and for $\varepsilon \cong 10^{-2}$ and $\varepsilon \cong 10^{-3}$. The micro scale shape functions are derived from the first two Bloch eigenmodes $\phi_{m}(y, \eta), m=1,2$, by collocation and orthogonalization (collocation set as before). We see that the convergence occurs even later and this procedure does not automatically improve the convergence. We conclude that in the gFEM based on the micro-scale shape functions $\mathcal{M}_{\varepsilon, B}^{\mu}$ derived by collocating the Bloch eigenmodes essential information of the microstructure is missing. In addition, once discretizing the unit-cell problem, one has to solve parameter dependent eigenvalue problems which is more costly than solving linear systems.

\section{References}

1. G. Allaire, M. Amar, Boundary Layer Tails in Periodic Homogenization, European Series in Applied and Industrial Mathematics, 1999.

2. I. Babuška, B. Anderson, P. J. Smith, K. Levin, Damage Analysis of Fiber Composites. Part I: Statistical Analysis on Fiber Scale, Preprint No.15, The Aeronautical Research Institute of Sweden (1998).

3. I. Babuška, A. K. Aziz, Survey lectures on the mathematical foundation of the finite element method, in The Mathematical Foundations of the Finite Element Method with Applications to Partial Differential Equations, A. K. Aziz, ed., Academic Press, New York (1973) 5-359.

4. I. Babuška, G. Caloz, J. Osborn, Special finite element methods for a class of second order elliptic problems with rough coefficients, SIAM J. Numer. Anal., 31 No. 4, (1994), 945-981. 
5. I. Babuška, B. Q. Guo, Regularity of the solution of elliptic problems with piecewise analytic data. Part 1: Boundary value problems for linear elliptic equation of second order, SIAM J. Math. Anal., 19 (1988), pp. 172-203.

6. I. Babuška and J.M. Melenk, The partition of unity Finite Element Method: Basic theory and applications Comp. Meth. Appl. Mech. Engg. 40 (1997) 727758.

7. N. Bakhvalov, G. Panasenko, Homogenization: Averaging Process in Periodic Media, Kluwer Publ., Dordrecht, 1989.

8. A. Bensoussan, J. L. Lions, G. Papanicolau, Asymptotic Analysis for Periodic Structures, North Holland, Amsterdam, 1978.

9. F. Bloch, Über die Quantenmechanik der Electronen in Kristallgittern, Z. Phys. 52 (1928), 555-600.

10. S.C. Brenner and L.R. Scott, The Mathematical Theory of Finite Element Methods, Texts in Applied Mathematics 15, Springer Verlag, New York, 1994.

11. J. Céa, Approximation variationelle des problèmes aux limites, Ann. Inst. Fourier (Grenoble) 2, 1964, 610-620.

12. P.G. Ciarlet, The Finite Element Method for Elliptic Problems, North-Holland Publishing Company, 1976.

13. D. Cioranescu and Patrizia Donato, An Introduction to homogenization, Oxford University Press (1999).

14. D. Cioranescu and Jeanine Saint Jean Paulin, Homogenization of Reticulated Structures, Springer Applied Mathematical Sciences (1999).

15. C. Conca, M. Vanninathan, Homogenization of periodic structures via Bloch decomposition, SIAM J. Appl. Math. Vol. 57, No. 6, (1997) 1639-1659.

16. P. J. Davis, Interpolation \& Approximation, Dover Publications, INC. New York, 1974.

17. I.S. Gradshteyn and I.M. Ryzhik, Table of Integrals, Series, and Producs, Corrected and enlarged edition. Wiley, New York, 1980.

18. W. Hackbusch, S. A. Sauter, Composite Finite Elements for the Approximation of PDEs on Domains with complicated Micro-Structures, Numer. Math. 75 (1997), 447-472.

19. W. Hackbusch and Stefan Sauter, Composite finite elements for problems containing small geometric details Part II: Implementation and numerical results, Computing and Visualization in Science 1 (1997), 15-25

20. T. Y. Hou, X. H. Wu, A Multiscale Finite Element Method for Elliptic Problems in Composite Materials and Porous Media J. Comput Phys., 134, (1997) 169189.

21. T. Hou, X.-H. Wu, Z. Cai, Convergence of a multiscale finite element method for elliptic problems with rapidly oscillating coefficients, to appear in Math. Comp.

22. Jonathan Aerospace materials corporation online brochure, Executive Summary, Wilmington, Ma USA (available via email from info@jamcorp.com).

23. G.E. Karniadakis, S.J. Sherwin, Spectral hp Element Methods for CFD, Oxford University Press, 1999.

24. C. Lage, Concept Oriented Design of Numerical Software, Technical Report 98-07, Seminar for Applied Mathematics, ETH Zürich http://www.sam.math.ethz.ch/Reports/prepr98.html/98-07.ps.Z.

25. P.D. Lax, A.N. Milgram, Parabolic Equations, volume 33 of Annals of Mathematics Studies, Princeton University Press, 1954, 167-190.

26. A.M. Matache, Spectral and $p$-Finite Elements for Problems with Microstructure, Doctoral Thesis, ETH Zürich 2000.

27. A.M. Matache and Ch. Schwab, Homogenization via $p$-FEM for Problems with Microstructure, Applied Numerical Mathematics, vol 33, Issue 1-4, May 2000, available also as Report 99-04, Seminar for Applied Mathematics, ETH Zürich, http://www.sam.math.ethz.ch/Reports/ prepr99.html/99-09.ps.Z.

28. A.M. Matache, C. Schwab, Two-Scale FEM for Homogenization Problems, in preparation.

29. A.M. Matache, I. Babuška and Ch. Schwab, Generalized p-FEM in Homogenization, Numerische Mathematik, 86 Issue 2 (2000), 319-375. 
30. J.M. Melenk, On n-widths for elliptic problems, Journal of Mathematical Analysis and Applications, Vol.247, No. 1, Jul 2000, pp. 272-289.

31. R. C. Morgan, I. Babuška, An approach for constructing families of homogenized equations for periodic media. I: An integral representation and its consequences, SIAM J. Math. Anal. Vol. 22, No. 1, pp.1-15, January 1991.

32. R. C. Morgan, I. Babuška, An approach for constructing families of homogenized equations for periodic media. II: Properties of the kernel, SIAM J. Math. Anal. Vol. 22, No. 1, pp.16-33, January 1991.

33. N. Neuss, W. Jäger, G. Wittum, Homogenization and Multigrid, Preprint 199804, SFB 359, University of Heidelberg.

34. M. Neuss-Radu, Boundary Layers in the Homogenization of Elliptic Problems, Doctoral Dissertation, Heidelberg University, 1999.

35. O. A. Oleinik, A.S. Shamaev, G. A. Yosifian, Mathematical Problems in Elasticity and Homogenization, North-Holland, 1992.

36. F.W.J. Olver, Asymptotics and Special Functions, Academic Press, 1974.

37. C. Schwab, $p$ - and hp- Finite Element Methods, Oxford University Press, 1998.

38. C. Schwab, A.M. Matache, High order generalized FEM for lattice materials, Proceedings of the 3rd European Conference on Numerical Mathematics and Advanced Applications, Finland, 1999, ed. by P. Neittaanmäki, T. Tiihonen and P. Tarvainen, World Scientific, Singapore, 2000.

39. C. Schwab, M. Suri, The $p$ and hp versions of the Finite Element Method for problems with boundary layers, Mathematics of Computation, Vol. 65, No. 216, (1996) 1403-1429.

40. Frank Stenger, Numerical Methods Based on Sinc and Analytic Functions, Springer Verlag, 1993. 

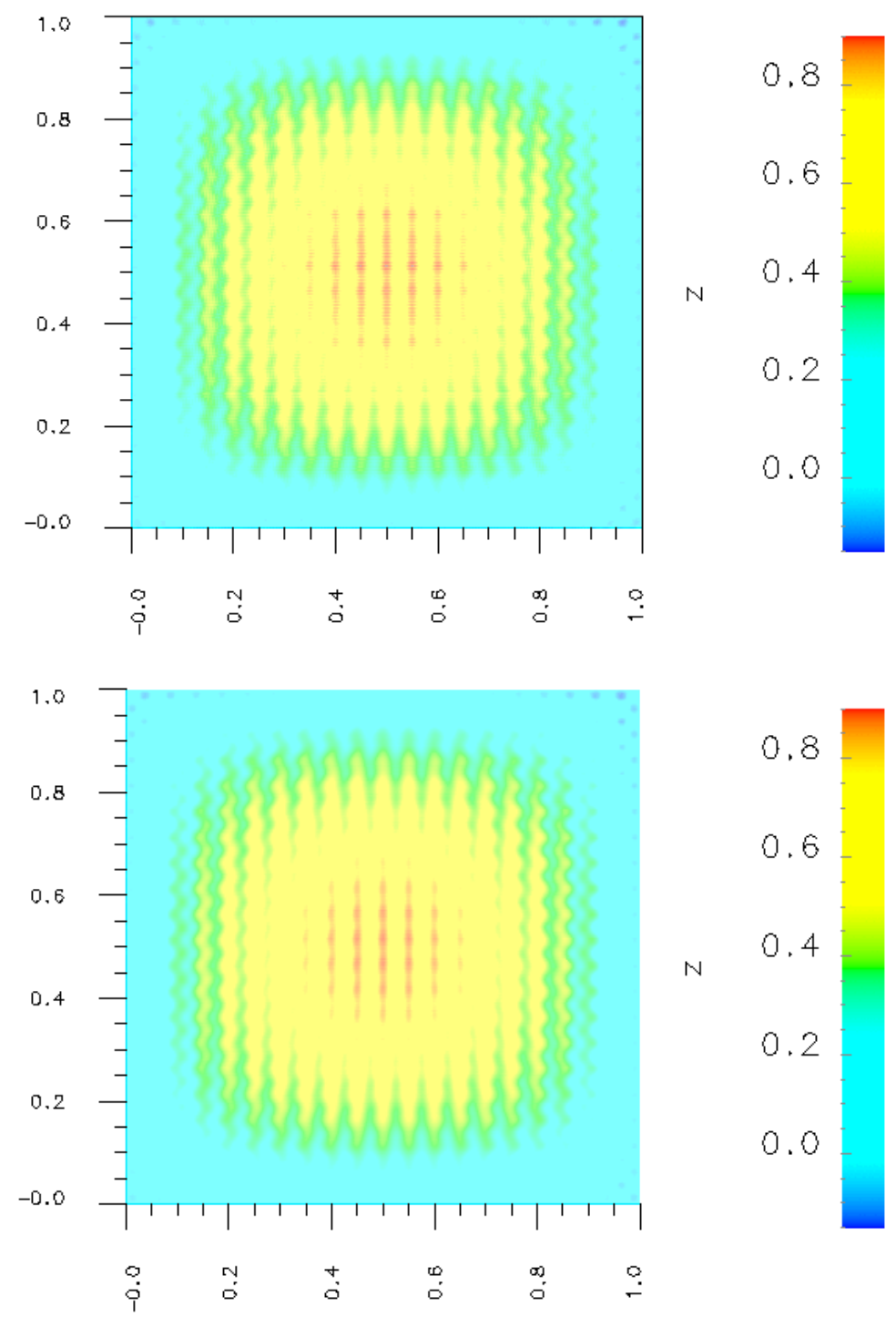

Fig. 8. The second component of the flux $a(x / \varepsilon) \nabla u^{\varepsilon}$ obtained by the generalized FEM based on oversampling (top) and by direct FEM computation (bottom); $\widehat{Q}=$ $(0,2 \pi)^{2}, \varepsilon=1 /(40 \pi)$. 

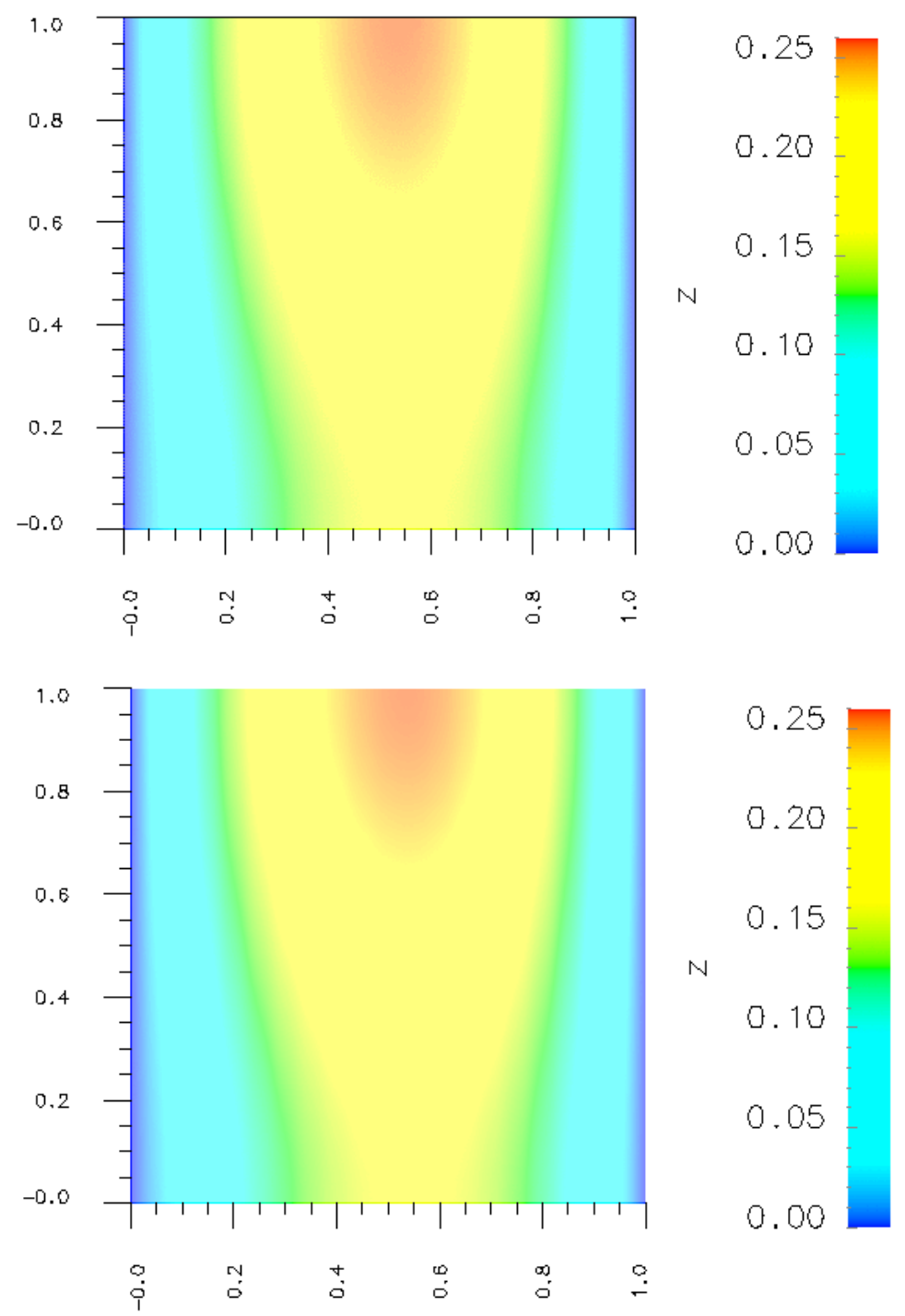

Fig. 9. The numerical solutions obtained by the generalized FEM based on oversampling (top) and by direct FEM computation (bottom); $\widehat{Q}=(0,2 \pi)^{2}, \varepsilon=1 /(40 \pi)$. 


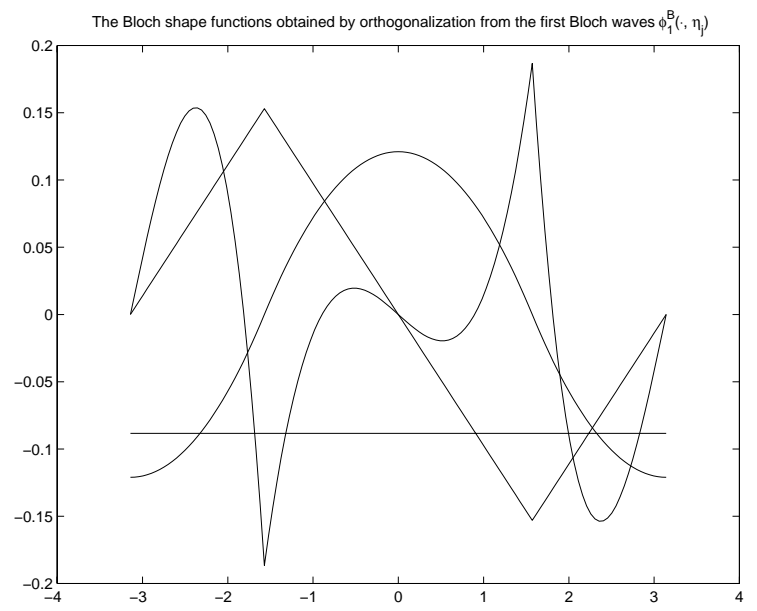

Fig. 10. The shape functions $\phi_{i}^{B}(\cdot)$ obtained by orthogonalization from the first Bloch eigenmode $\phi_{1}\left(\cdot ; \eta_{j}\right)$ sampled at $\eta_{j}=10^{-j}, j=1,2, \ldots, 10$
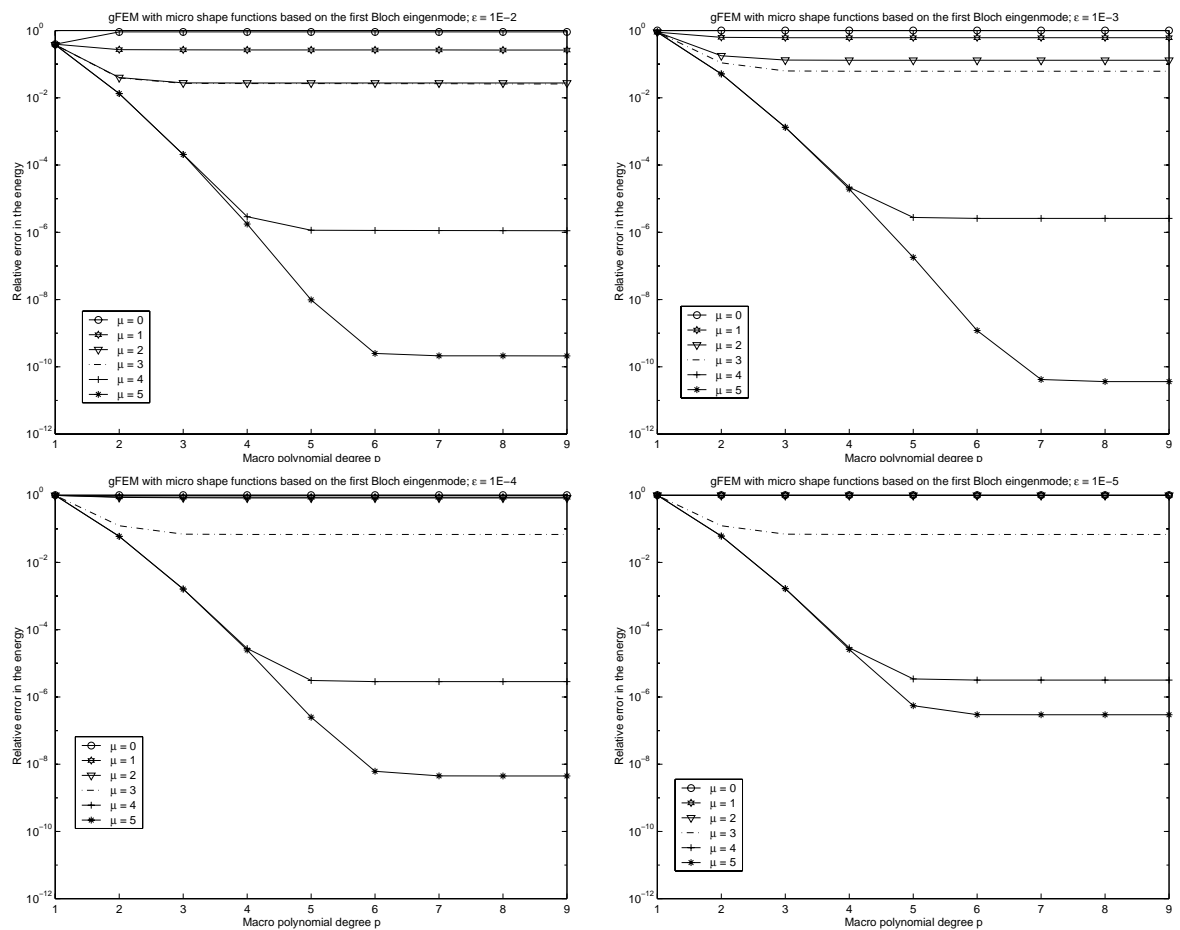

Fig. 11. Relative error for the energy versus $p$ with $\mathcal{M}_{\varepsilon}^{\mu}=\mathcal{M}_{\varepsilon, B}^{\mu}$ obtained from the collocated first Bloch eigenmode; $f(x)=\exp (x)$. 

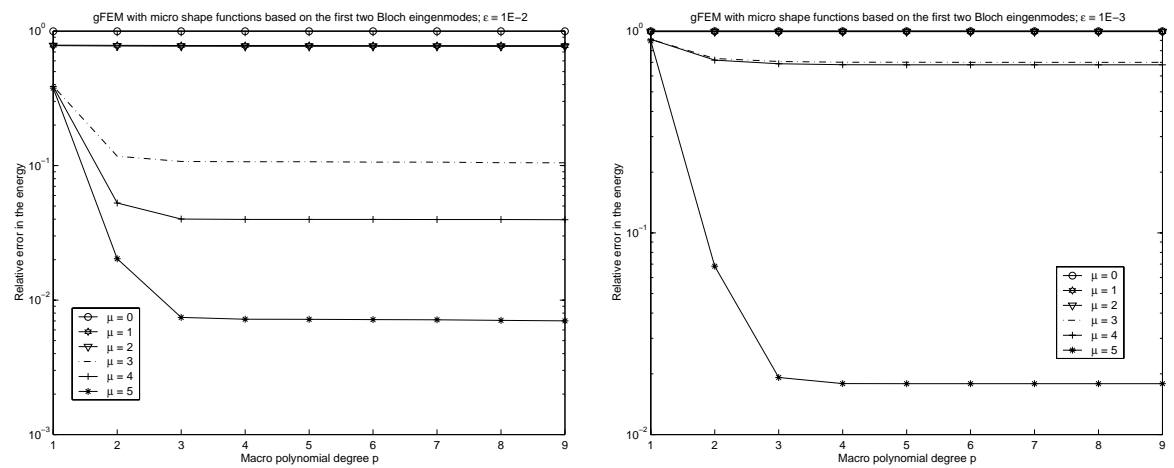

Fig. 12. Relative error for the energy versus $p$ with $\mathcal{M}_{\varepsilon}^{\mu}=\mathcal{M}_{\varepsilon, B}^{\mu}$ obtained from the first two collocated Bloch eigenmodes $\phi_{m}(\cdot, \eta), m=1,2 ; f(x)=\exp (x)$. 